\title{
Deterministic homogenization of integral functionals with convex integrands
}

\author{
Gabriel Nguetseng, Hubert Nnang and Jean Louis Woukeng
}

\begin{abstract}
In order to widen the scope of the applications of deterministic homogenization, we consider here the homogenization problem for a family of integral functionals. The homogenization procedure tending to be classical, the choice focused on the convex integral functionals is made just to simplify the presentation of the paper. We use a new approach based on the Stepanov type spaces, which approach allows us to solve various problems such as the almost periodic homogenization problem and others without resorting to additional assumptions. We then apply it to obtain a general homogenization result and then we provide a number of physical applications of the result. The convergence method used falls within the scope of two-scale convergence.
\end{abstract}

Mathematics Subject Classification (2000). 35B40, 45G10, 46J10.

Keywords. Homogenization, Convex integrands, Homogenization algebras, Sigma-convergence.

\section{Introduction}

We study the asymptotic behaviour (as $0<\varepsilon \rightarrow 0$ ) of the sequence of solutions to the problems

$$
\min \left\{F_{\varepsilon}(v): v \in W_{0}^{1, p}\left(\Omega ; \mathbb{R}^{n}\right)\right\}
$$

where the functional $F_{\varepsilon}$ is defined on $W_{0}^{1, p}\left(\Omega ; \mathbb{R}^{n}\right)$ by

$$
F_{\varepsilon}(v)=\int_{\Omega} f\left(x, \frac{x}{\varepsilon}, D v(x)\right) d x\left(v \in W_{0}^{1, p}\left(\Omega ; \mathbb{R}^{n}\right)\right),
$$

$\Omega$ being a bounded open set in $\mathbb{R}^{N}$ (integers $n, N \geq 1$ ), $D$ denoting the gradient operator in $\Omega$, and the function $f: \mathbb{R}^{N} \times \mathbb{R}^{N} \times \mathbb{R}^{n N} \rightarrow[0,+\infty)$ satisfying the following conditions:

$\left(\mathrm{H}_{1}\right)$ There exist a continuous positive function $\omega: \mathbb{R} \rightarrow \mathbb{R}_{+} \equiv[0,+\infty)$ with $\omega(0)=0$, and a function $a \in L_{\mathrm{loc}}^{1}\left(\mathbb{R}_{y}^{N}\right)$ such that 


$$
\left|f(x, y, \lambda)-f\left(x^{\prime}, y, \lambda\right)\right| \leq \omega\left(\left|x-x^{\prime}\right|\right)(a(y)+f(x, y, \lambda))
$$

for all $x, x^{\prime} \in \mathbb{R}^{N}, \lambda \in \mathbb{R}^{n N}$ and for almost all $y \in \mathbb{R}^{N}$,

$\left(\mathrm{H}_{2}\right) f(x, \cdot, \lambda)$ is measurable for all $(x, \lambda) \in \mathbb{R}^{N} \times \mathbb{R}^{n N}$,

$\left(\mathrm{H}_{3}\right) f(x, y, \cdot)$ is strictly convex for almost all $y \in \mathbb{R}^{N}$ and for all $x \in \mathbb{R}^{N}$,

$\left(\mathrm{H}_{4}\right)$ There exist three constants $p>1$ and $c_{1}, c_{2}>0$ such that

$$
c_{1}|\lambda|^{p} \leq f(x, y, \lambda) \leq c_{2}\left(1+|\lambda|^{p}\right)
$$

for all $(x, \lambda) \in \mathbb{R}^{N} \times \mathbb{R}^{n N}$ and for almost all $y \in \mathbb{R}^{N}$.

Since the function $f(x, y, \cdot)$ is convex, it comes from (1.3) [see in particular the right-hand side of the inequality in (1.3)] that

$$
\begin{aligned}
& \left|f(x, y, \lambda)-f\left(x, y, \lambda^{\prime}\right)\right| \leq c_{2}\left(1+|\lambda|^{p-1}+\left|\lambda^{\prime}\right|^{p-1}\right)\left|\lambda-\lambda^{\prime}\right| \\
& \quad \text { for all } x \in \mathbb{R}^{N}, \lambda, \lambda^{\prime} \in \mathbb{R}^{n N} \text { and for almost all } y \in \mathbb{R}^{N} .
\end{aligned}
$$

Consequently, for any fixed $\varepsilon>0$ and for $w \in L^{p}\left(\Omega ; \mathbb{R}^{n N}\right)$, the function $x \mapsto f(x, x / \varepsilon, w(x))$ of $\Omega$ into $\mathbb{R}_{+}$(denoted by $f^{\varepsilon}(\cdot, \cdot, w)$ ), is well defined and lies in $L^{1}(\Omega)$ (see [21]), with

$$
c_{1}\|w\|_{L^{p}(\Omega)^{n N}}^{p} \leq\left\|f^{\varepsilon}(\cdot, \cdot, w)\right\|_{L^{1}(\Omega)} \leq c_{2}^{\prime}\left(1+\|w\|_{L^{p}(\Omega)^{n N}}^{p}\right)
$$

where $c_{2}^{\prime}=c_{2} \max (1,|\Omega|)$ with $|\Omega|=\int_{\Omega} d x$. Indeed due to the inequalities (1.2) and (1.4) the mapping $\left(x, x^{\prime}, y\right) \mapsto f\left(x, y, w\left(x^{\prime}\right)\right.$ ) (for any fixed $w$ in $\left.\mathcal{C}\left(\bar{\Omega} ; \mathbb{R}^{n N}\right)\right)$ from $\bar{\Omega} \times \bar{\Omega} \times \mathbb{R}_{y}^{N}$ to $\mathbb{R}_{+}$, lies in $\mathcal{C}\left(\bar{\Omega} \times \bar{\Omega} ; L^{\infty}\left(\mathbb{R}_{y}^{N}\right)\right)$, and so one can naturally define the trace $(x, y) \mapsto f(x, y, w(x))$ of $\bar{\Omega} \times \mathbb{R}_{y}^{N}$ into $\mathbb{R}_{+}$as follows: $f(x, y, w(x))=\left.f\left(x, y, w\left(x^{\prime}\right)\right)\right|_{x^{\prime}=x}$, which belongs to $\mathcal{C}\left(\bar{\Omega} ; L^{\infty}\left(\mathbb{R}_{y}^{N}\right)\right)$. Therefore, due to [20, Proposition 1.5], one can easily define the trace function $x \mapsto f(x, x / \varepsilon, w(x))$, of $\Omega$ into $\mathbb{R}_{+}$, as an element of $L^{\infty}\left(\Omega ; \mathbb{R}_{+}\right)$; and by density, owing to [21], the function $x \mapsto f(x, x / \varepsilon, w(x))$ (for $w \in L^{p}\left(\Omega ; \mathbb{R}^{n N}\right)$ ) is well defined and lies in $L^{1}(\Omega)$. Hence, assuming the vector space $W_{0}^{1, p}\left(\Omega ; \mathbb{R}^{n}\right)$ to be endowed with the norm $\|v\|_{W_{0}^{1, p}(\Omega)^{n}}=\|D v\|_{L^{p}(\Omega)^{n N}} \quad$ (which makes it a Banach space), we have in particular

$$
c_{1}\|v\|_{W_{0}^{1, p}(\Omega)^{n}}^{p} \leq\left\|f^{\varepsilon}(\cdot, \cdot, D v)\right\|_{L^{1}(\Omega)} \leq c_{2}^{\prime}\left(1+\|v\|_{W_{0}^{1, p}(\Omega)^{n}}^{p}\right),
$$

which allows us to justify the definition (1.1). Therefore, using together the inequality (1.4) (which implies the continuity of the functional $F_{\varepsilon}$ ) with the strict convexity of $F_{\varepsilon}\left(\right.$ see $\left.\left(\mathrm{H}_{3}\right)\right)$ and the left-hand side of the inequality in (1.5) (which means the coercivity of $F_{\varepsilon}$ ), we deduce $[10,24]$ the existence of a unique $u_{\varepsilon} \in W_{0}^{1, p}\left(\Omega ; \mathbb{R}^{n}\right)$ solution to the minimization problem

$$
F_{\varepsilon}\left(u_{\varepsilon}\right)=\min _{v \in W_{0}^{1, p}\left(\Omega ; \mathbb{R}^{n}\right)} F_{\varepsilon}(v) .
$$

Under an abstract structure assumption (to be specified later), the homogenization of functionals $F_{\varepsilon}$ amounts to finding a homogenized functional $F$ such that the sequence of minimizers $u_{\varepsilon}$ converges to a limit $\mathbf{u}$, which turns out to be the minimizer of $F$.

From a physical point of view, e.g., in elasticity theory, the term $F_{\varepsilon}(v)$ can be viewed as the energy under a deformation $v$ of an elastic body whose 
microstructure behaves realistically. In the case where the microstructure has a periodic behaviour, the functionals $F_{\varepsilon}$ have been attracted the attention of a great number of researchers. We refer, e.g., to $[1,2,5,11,24]$. In [5], the functionals of the type (1.1) have been studied by Braides under assumptions $\left(\mathrm{H}_{1}\right)-\left(\mathrm{H}_{4}\right)$. Recently, Baia and Fonseca [2] have studied this problem under hypothesis $\left(\mathrm{H}_{4}\right)$ and requiring continuity on $(y, \lambda)$ and measurability in $x$. It is to be noted that in $[1,11,24]$, the authors have considered functionals of the form

$$
\int_{\Omega} f\left(\frac{x}{\varepsilon}, D v(x)\right) d x .
$$

The main references for functionals of type (1.7) are Marcellini [17] and Carbone-Sbordone [7].

Apart from [1], all the works cited above have two common points : (1) they have been studied under the periodicity hypothesis on the fast variable $y$; (2) the convergence method used is that of $\Gamma$-convergence [12]. In [1], under a periodic hypothesis, and a differentiability hypothesis on $f$ with respect to $\lambda$, Allaire recovers some explicit results via the technique of two-scale convergence. Concerning the results beyond the periodic setting, Kozlov [15] was the first to prove an homogenization result for functionals of the form (1.7), with $f$ being a quadratic form in its second occurrence and almost periodic in the first one. Later on, Braides [6], using the $\Gamma$-convergence techniques, studied functionals (1.7) under the almost periodicity assumption with respect to the fast variable $y$ and the quasiconvexity hypothesis on $f$ with respect to the second variable.

Our goal in this paper is to study the asymptotic behaviour of $F_{\varepsilon}$ under an abstract assumption covering a wide range of concrete behaviours such as the periodicity, the almost periodicity, the convergence at infinity, and many more besides. We present here a new approach to solve deterministic homogenization problems. This new approach is based on the generalized Stepanov type spaces, and widely opens the scope of applications of our result, Theorem 3.1, as can be easily seen in Sect. 4. In particular this approach allows us to work out the almost periodic homogenization problem without any further assumption on the function $f$ as is usually the case in all the previous works dealing with deterministic homogenization theory; see for instance [21,22]. The homogenization approach followed here is the $\Sigma$-convergence method proceeding from two-scale convergence ideas and use of the so-called homogenization algebras; see [18] for more details.

The paper is framed as follows. Section 2 deals with notations and preliminary results. In Sect. 3, we study the homogenization of problem (1.6) under an abstract hypothesis on $f(x, y, \lambda)$ (for fixed $x, \lambda$ ). Finally, in Sect. 4 , we solve some concrete homogenization problems for (1.6).

Unless otherwise specified, vector spaces throughout are assumed to be complex vector spaces, and scalar functions are assumed to take values in $\mathbb{C}$ (the complex). This permits us to make use of basic tools provided by the 
classical Banach algebras theory. For basic concepts and notation about integration theory we refer to [4]. We shall always assume that the numerical spaces $\mathbb{R}^{m}$ and their open sets are each equipped with the Lebesgue measure.

\section{Notations and preliminary results}

\subsection{Homogenization algebras: an overview of basic results}

We recall that $\mathcal{B}\left(\mathbb{R}_{y}^{N}\right)$ denotes the space of bounded continuous complex functions on $\mathbb{R}_{y}^{N}$ (the space $\mathbb{R}^{N}$ of variables $y=\left(y_{1}, \ldots, y_{N}\right)$ ). It is well known that $\mathcal{B}\left(\mathbb{R}_{y}^{N}\right)$ with the sup norm and the usual algebra operations is a commutative $\mathcal{C}^{*}$-algebra with identity (the involution is here the usual one of complex conjugation).

Throughout the present Sect. 2, $A$ denotes a separable closed subalgebra of the Banach algebra $\mathcal{B}\left(\mathbb{R}_{y}^{N}\right)$. Furthermore, we assume that $A$ contains the constants, $A$ is stable under complex conjugation (i.e., the complex conjugate, $\bar{u}$, of any $u \in A$ still lies in $A$ ), and finally, $A$ has the following property : for any $u \in A$, we have $u^{\varepsilon} \rightarrow M(u)$ in $L^{\infty}\left(\mathbb{R}_{x}^{N}\right)$-weak $*$ as $\varepsilon \rightarrow 0(\varepsilon>0)$, where $M(u) \in \mathbb{C}$ and

$$
u^{\varepsilon}(x)=u\left(\frac{x}{\varepsilon}\right) \quad\left(x \in \mathbb{R}^{N}\right) .
$$

The complex mapping $u \mapsto M(u)$ on $A$, denoted by $M$, is a nonnegative continuous linear form with $M(1)=1$; so that $M$ is a mean value (see [19]).

$A$ is called an $H$-algebra ( $H$ stands for homogenization). It is clear that $A$ is a commutative $\mathcal{C}^{*}$-algebra with identity. We denote by $\Delta(A)$ the spectrum of $A$ and by $\mathcal{G}$ the Gelfand transformation on $A$. For the benefit of the reader it is worth recalling that $\Delta(A)$ is the set of all nonzero multiplicative linear forms on $A$, and $\mathcal{G}$ is the mapping of $A$ into $\mathcal{C}(\Delta(A))$ such that $\mathcal{G}(u)(s)=\langle s, u\rangle$ $\left(s \in \Delta(A)\right.$ ), where $\langle$,$\rangle denotes the duality pairing between A^{\prime}$ (the topological dual of $A$ ) and $A$. The appropriate topology on $\Delta(A)$ is the relative weak * topology on $A^{\prime}$. So topologized, $\Delta(A)$ is a metrizable compact space, and the Gelfand transformation is an isometric isomorphism of the $\mathcal{C}^{*}$-algebra $A$ onto the $\mathcal{C}^{*}$-algebra $\mathcal{C}(\Delta(A))$. See, e.g., [16] for further details concerning the Banach algebras theory. Since the mean value $M$ is a nonnegative continuous linear functional on $A$ with $M(1)=1$, this provides us with a linear nonnegative functional $\psi \mapsto M_{1}(\psi)=M\left(\mathcal{G}^{-1}(\psi)\right)$ defined on $\mathcal{C}(\Delta(A))=\mathcal{G}(A)$ which is clearly bounded. Therefore, by the Riesz-Markov theorem, $M_{1}(\psi)$ is representable by integration with respect to a Radon measure $\beta$ (of total mass 1$)$ in $\Delta(A)$, called the $M$-measure for $A$. It is evident that we have $M(u)=\int_{\Delta(A)} \mathcal{G}(u) d \beta$ for $u \in A$.

Before we go any further, it seems necessary to render more explicit the notion of the spectrum of an $H$-algebra in some well-known cases. If $A$ is the periodic $H$-algebra $\mathcal{C}_{\text {per }}(Y)$ of $Y$-periodic continuous functions on $\mathbb{R}^{N}$, then $\Delta(A)$ can be identified with the period $Y=[-1 / 2,1 / 2]^{N}$. Let $\mathcal{R}$ be a countable subgroup of $\mathbb{R}^{N}$. Let $A P_{\mathcal{R}}\left(\mathbb{R}^{N}\right)$ denote the algebra of functions on $\mathbb{R}^{N}$ that are uniformly approximated by finite linear combinations of the functions 
in the set $\left\{\gamma_{k}: k \in \mathcal{R}\right\}$ where $\gamma_{k}$ is defined by $\gamma_{k}(y)=\exp (2 i \pi k \cdot y)\left(y \in \mathbb{R}^{N}\right)$. It is known that $A P_{\mathcal{R}}\left(\mathbb{R}^{N}\right)$ is an $H$-algebra [18, Example 2.2] and its spectrum $\Delta\left(A P_{\mathcal{R}}\left(\mathbb{R}^{N}\right)\right)$ is a compact topological group homeomorphic to the dual group $\widehat{\mathcal{R}}$ of $\mathcal{R}$ consisting of the characters $\gamma_{k}(k \in \mathcal{R})$ of $\mathbb{R}^{N}$; see [20, Propositions 2.2 and 2.6] for details.

The partial derivative of index $i(1 \leq i \leq N)$ on $\Delta(A)$ is defined to be the mapping $\partial_{i}=\mathcal{G} \circ D_{y_{i}} \circ \mathcal{G}^{-1}$ (usual composition) of $\mathcal{D}^{1}(\Delta(A))=\{\varphi \in \mathcal{C}(\Delta(A))$ : $\left.\mathcal{G}^{-1}(\varphi) \in A^{1}\right\}$ into $\mathcal{C}(\Delta(A))$, where $A^{1}=\left\{\psi \in \mathcal{C}^{1}\left(\mathbb{R}_{y}^{N}\right): \psi, \frac{\partial \psi}{\partial y_{i}} \in A(1 \leq i \leq\right.$ $N)\}, D_{y_{i}}=\frac{\partial}{\partial y_{i}}$. Higher order derivatives can be defined analogously (see [18]). Now, let $A^{\infty}$ be the space of $\psi \in \mathcal{C}^{\infty}\left(\mathbb{R}_{y}^{N}\right)$ such that $D_{y}^{\alpha} \psi=\frac{\partial^{|\alpha|} \psi}{\partial y_{1}^{\alpha_{1}} \ldots \partial y_{N}^{\alpha_{N}}} \in A$ for every multi-index $\alpha=\left(\alpha_{1}, \ldots, \alpha_{N}\right) \in \mathbb{N}^{N}$, and let $\mathcal{D}(\Delta(A))=\{\varphi \in \mathcal{C}(\Delta(A))$ : $\left.\mathcal{G}^{-1}(\varphi) \in A^{\infty}\right\}$. Endowed with a suitable locally convex topology (see [18]), $A^{\infty}$ (respectively, $\mathcal{D}(\Delta(A))$ ) is a Fréchet space and further, $\mathcal{G}$ viewed as defined on $A^{\infty}$ is a topological isomorphism of $A^{\infty}$ onto $\mathcal{D}(\Delta(A))$.

By a distribution on $\Delta(A)$ we mean any continuous linear form on $\mathcal{D}(\Delta(A))$. The space of all distributions on $\Delta(A)$ is then the dual, $\mathcal{D}^{\prime}(\Delta(A))$, of $\mathcal{D}(\Delta(A))$. We endow $\mathcal{D}^{\prime}(\Delta(A))$ with the strong dual topology. The following result permits us to view the space $L^{p}(\Delta(A))(1 \leq p \leq \infty)$ as a subspace of $\mathcal{D}^{\prime}(\Delta(A))$ and so will allows us to define the Sobolev type spaces associated to the spectrum of an $H$-algebra.

Proposition 2.1. Let $A$ be an $H$-algebra. Assume $A$ is translation invariant, that is, $\tau_{a} u \in A$ for any $a \in \mathbb{R}^{N}$, where $\tau_{a} u \equiv u(\cdot-a)$, and moreover that each element of $A$ is uniformly continuous. Then the space $A^{\infty}$ is dense in $A$.

Proof. Let $u \in A$; then for all $\varphi \in \mathcal{D}\left(\mathbb{R}^{N}\right)$ we have $\varphi * u \in \mathcal{C}^{\infty}\left(\mathbb{R}^{N}\right)$ where $\varphi * u$ stands for the product of convolution defined by $(\varphi * u)(y)=\int \varphi(\zeta) u(y-\zeta) d \zeta$ $\left(y \in \mathbb{R}^{N}\right)$. Now, since $u$ and $\varphi$ are uniformly continuous, there exist, for every $\varepsilon>0$, some $y_{1}, \ldots, y_{n} \in \mathbb{R}^{N}$ and some positive real numbers $\mu_{1}, \ldots, \mu_{n}$ such that

$$
\sup _{x \in \mathbb{R}^{N}}\left|\int u(x-y) \varphi(y) d y-\sum_{i=1}^{n} u\left(x-y_{i}\right) \varphi\left(y_{i}\right) \mu_{i}\right|<\varepsilon
$$

i.e.,

$$
\left\|\varphi * u-\sum_{i=1}^{n} \varphi\left(y_{i}\right) \mu_{i} \tau_{y_{i}} u\right\|_{\infty}<\varepsilon
$$

where $\tau_{y_{i}} u(x)=u\left(x-y_{i}\right)$ for $x \in \mathbb{R}^{N}$. But $A$ is translation invariant, hence $\tau_{y_{i}} u \in A$ and so $\sum_{i=1}^{n} \tau_{y_{i}} u \varphi\left(y_{i}\right) \mu_{i} \in A$, and therefore $\varphi * u \in A, A$ being closed with the sup norm topology. Since $D^{\alpha}(\varphi * u)=D^{\alpha} \varphi * u$ for all $\alpha \in \mathbb{N}^{N}$, we conclude that $D^{\alpha}(\varphi * u) \in A$, and so, $\varphi * u \in A^{\infty}$.

Now let $\left(\theta_{n}\right)_{n \geq 1}$ be a mollifier on $\mathbb{R}^{N}$, i.e. $\left(\theta_{n}\right)_{n \geq 1} \subset \mathcal{D}\left(\mathbb{R}^{N}\right)$ with $\theta_{n} \geq 0$, $\int \theta_{n}(y) d y=1, \theta_{n}$ has support contained in $\frac{1}{n} \bar{B}_{N}$, where $\bar{B}_{N}$ is the closed unit ball in $\mathbb{R}^{N}$. Set $u_{n}=\theta_{n} * u(n \geq 1)$; clearly $u_{n} \in A^{\infty}$ and $u_{n}(x)-u(x)=$ 
$\int(u(x-y)-u(x)) \theta_{n}(y) d y$, hence

$$
\left\|u_{n}-u\right\|_{\infty} \leq \sup _{x \in \mathbb{R}^{N}} \sup _{|y| \leq \frac{1}{n}}|u(x-y)-u(x)| .
$$

But $u$ is uniformly continuous and bounded since $A$ is an $H$-algebra. One deduces that $\sup _{x \in \mathbb{R}^{N}} \sup _{|y| \leq \frac{1}{n}}|u(x-y)-u(x)| \rightarrow 0$ as $n \rightarrow \infty$, i.e. $u_{n} \rightarrow u$ in $A$ as $n \rightarrow \infty$. The proposition follows thereby.

It will be an easy exercise (left to the reader) to see that all the $H$-algebras encountered in this paper are translation invariant. Any $H$-algebra $A$ verifying the property that $A^{\infty}$ is dense in $A$ will be termed of class $\mathcal{C}^{\infty}$. The above result provides us with a large class of $H$-algebras of class $\mathcal{C}^{\infty}$.

Proposition 2.1 is equivalent to say that $\mathcal{D}(\Delta(A))\left(=\mathcal{G}\left(A^{\infty}\right)\right)$ is dense in $\mathcal{C}(\Delta(A))(=\mathcal{G}(A))$, so that one can easily see that $L^{p}(\Delta(A)) \subset \mathcal{D}^{\prime}(\Delta(A))$ $(1 \leq p \leq \infty)$ with continuous embedding. Hence we may define the Sobolev space

$$
W^{1, p}(\Delta(A))=\left\{u \in L^{p}(\Delta(A)): \partial_{i} u \in L^{p}(\Delta(A))(1 \leq i \leq N)\right\}(1 \leq p<\infty)
$$

where the derivative $\partial_{i} u$ is taken in the distribution sense on $\Delta(A)$ (exactly as the Schwartz derivative in the classical case). This is a Banach space with the norm

$$
\|u\|_{W^{1, p}(\Delta(A))}=\left(\|u\|_{L^{p}(\Delta(A))}^{p}+\sum_{i=1}^{N}\left\|\partial_{i} u\right\|_{L^{p}(\Delta(A))}^{p}\right)^{\frac{1}{p}}\left(u \in W^{1, p}(\Delta(A))\right) .
$$

However, in practice the appropriate space is not $W^{1, p}(\Delta(A))$ but its closed subspace

$$
W^{1, p}(\Delta(A)) / \mathbb{C}=\left\{u \in W^{1, p}(\Delta(A)): \int_{\Delta(A)} u(s) d \beta(s)=0\right\}
$$

equipped with the seminorm

$$
\|u\|_{W^{1, p}(\Delta(A)) / \mathbb{C}}=\left(\sum_{i=1}^{N}\left\|\partial_{i} u\right\|_{L^{p}(\Delta(A))}^{p}\right)^{\frac{1}{p}} \quad\left(u \in W^{1, p}(\Delta(A)) / \mathbb{C}\right) .
$$

Unfortunately, the space $W^{1, p}(\Delta(A)) / \mathbb{C}$ is in general nonseparate and noncomplete. We introduce the separated completion, $W_{\#}^{1, p}(\Delta(A))$, of $W^{1, p}(\Delta(A)) / \mathbb{C}$, and the canonical mapping $J$ of $W^{1, p}(\Delta(A)) / \mathbb{C}$ into its separated completion. For $1<p<\infty, W_{\#}^{1, p}(\Delta(A))$ is a reflexive Banach space and $W_{\#}^{1,2}(\Delta(A))$ is a Hilbert space. Furthermore, as pointed out in [18], the distribution derivative $\partial_{i}$ viewed as a mapping of $W^{1, p}(\Delta(A)) / \mathbb{C}$ into $L^{p}(\Delta(A))$ extends to a unique continuous linear mapping, still denoted by $\partial_{i}$, of $W_{\#}^{1, p}(\Delta(A))$ into $L^{p}(\Delta(A))$ such that $\partial_{i} J(v)=\partial_{i} v$ for $v \in W^{1, p}(\Delta(A)) / \mathbb{C}$ and

$$
\|u\|_{W_{\#}^{1, p}(\Delta(A))}=\left(\sum_{i=1}^{N}\left\|\partial_{i} u\right\|_{L^{p}(\Delta(A))}^{p}\right)^{\frac{1}{p}} \text { for } u \in W_{\#}^{1, p}(\Delta(A)) .
$$


We will now recall the notion of $\Sigma$-convergence in $L^{p}(1 \leq p<\infty)$ in the present context. Before that, let us state another definition: the letter $E$ throughout will stand for any subset of positive real numbers admitting 0 as accumulation point. When $E$ is an ordinary sequence of reals $\left(\varepsilon_{n}\right)_{n \in \mathbb{N}}$ with $0<\varepsilon_{n} \leq 1$ and $\varepsilon_{n} \rightarrow 0$ as $n \rightarrow \infty$, it will be referred to as a fundamental sequence.

Definition 2.1. A sequence $\left(u_{\varepsilon}\right)_{\varepsilon \in E} \subset L^{p}(\Omega)$ is said to :

(i) weakly $\Sigma$-converge in $L^{p}(\Omega)$ to some $u_{0} \in L^{p}(\Omega \times \Delta(A))$ if as $E \ni \varepsilon \rightarrow 0$,

$$
\int_{\Omega} u_{\varepsilon}(x) \psi^{\varepsilon}(x) d x \rightarrow \iint_{\Omega \times \Delta(A)} u_{0}(x, s) \widehat{\psi}(x, s) d x d \beta(s)
$$

for all $\psi \in L^{p^{\prime}}(\Omega ; A)\left(\frac{1}{p^{\prime}}=1-\frac{1}{p}\right)$ where $\psi^{\varepsilon}(x)=\psi\left(x, \frac{x}{\varepsilon}\right)$ and $\widehat{\psi}(x, \cdot)=$ $\mathcal{G}(\psi(x, \cdot))$ a.e. in $x \in \Omega$;

(ii) strongly $\Sigma$-converge in $L^{p}(\Omega)$ to some $u_{0} \in L^{p}(\Omega \times \Delta(A))$ if the following property is verified : $\left\{\begin{array}{l}\text { Given } \eta>0 \text { and } v \in L^{p}(\Omega ; A) \text { with }\left\|u_{0}-\widehat{v}\right\|_{L^{p}(\Omega \times \Delta(A))} \leq \frac{\eta}{2} \text {, there } \\ \text { is some } \alpha>0 \text { such that }\left\|u_{\varepsilon}-v^{\varepsilon}\right\|_{L^{p}(\Omega)} \leq \eta \text { provided } E \ni \varepsilon \leq \alpha .\end{array}\right.$

Remark 2.1. The existence of such $v$ 's as in (ii) above results from the density of $L^{p}(\Omega ; \mathcal{C}(\Delta(A)))$ in $L^{p}\left(\Omega ; L^{p}(\Delta(A))\right)$.

We will briefly express weak and strong $\Sigma$-convergence by writing $u_{\varepsilon} \rightarrow$ $u_{0}$ in $L^{p}(\Omega)$-weak $\Sigma$ and $u_{\varepsilon} \rightarrow u_{0}$ in $L^{p}(\Omega)$-strong $\Sigma$, respectively.

For the main results regarding $\Sigma$-convergence, we will draw the reader's attention to [18]. However, we recall below one fundamental result and the notion of proper $H$-algebra which play crucial roles in $\Sigma$-convergence theory.

Theorem 2.2. Let $1<p<\infty$. Given a fundamental sequence $E$ and a sequence $\left(u_{\varepsilon}\right)_{\varepsilon \in E}$ which is bounded in $L^{p}(\Omega)$, a subsequence $E^{\prime}$ can be extracted from $E$ such that the sequence $\left(u_{\varepsilon}\right)_{\varepsilon \in E^{\prime}}$ weakly $\Sigma$-converges in $L^{p}(\Omega)$.

Proof. Set $L_{\varepsilon}(f)=\int_{\Omega} u_{\varepsilon} f^{\varepsilon} d x \quad\left(f \in L^{p^{\prime}}(\Omega ; A)\right)$, where $f^{\varepsilon} \in L^{p^{\prime}}(\Omega)$ is defined by $f^{\varepsilon}(x)=f(x, x / \varepsilon), x \in \Omega$. Then

$$
\left|L_{\varepsilon}(f)\right| \leq c\|f\|_{L^{p^{\prime}}(\Omega ; A)} \quad(\varepsilon \in E),
$$

hence $\left(L_{\varepsilon}(f)\right)_{\varepsilon \in E}$ is bounded in $\mathbb{C}$ and therefore, there exist a subsequence $E^{\prime}(f)$ and a complex number $L(\widehat{f})(\widehat{f}=\mathcal{G}(f), \mathcal{G}$ the Gelfand transformation on $A$ ) such that, as $E^{\prime}(f) \ni \varepsilon \rightarrow 0$, one has $L_{\varepsilon}(f) \rightarrow L(\widehat{f})$. Using the separability of $L^{p^{\prime}}(\Omega ; A)$ (note that $A$ is separable) and the diagonal process, one gets the existence of a subsequence $E^{\prime}$ from $E$ such that, as $E^{\prime} \ni \varepsilon \rightarrow 0$,

$$
L_{\varepsilon}(f) \rightarrow L(\widehat{f}) \text { for any } f \in L^{p^{\prime}}(\Omega ; A) .
$$

But one also has the inequality $\left|L_{\varepsilon}(f)\right| \leq c\left\|f^{\varepsilon}\right\|_{L^{p^{\prime}}(\Omega)}$ and further, as $E^{\prime} \ni \varepsilon \rightarrow$ $0,\left\|f^{\varepsilon}\right\|_{L^{p^{\prime}}(\Omega)} \rightarrow\|\widehat{f}\|_{L^{p^{\prime}}(\Omega \times \Delta(A))}$ (see e.g. [18]), so that the following holds:

$$
|L(\widehat{f})| \leq c\|\widehat{f}\|_{L^{p^{\prime}}(\Omega \times \Delta(A))} .
$$


Hence the linear form $L: \widehat{f} \mapsto L(\widehat{f})$, from $L^{p^{\prime}}(\Omega ; \mathcal{C}(\Delta(A)))$ to $\mathbb{C}$, extends by continuity to an element of $L^{p^{\prime}}(\Omega \times \Delta(A))$ (because $L^{p^{\prime}}(\Omega ; \mathcal{C}(\Delta(A)))$ is dense in $\left.L^{p^{\prime}}(\Omega \times \Delta(A))\right)$. Therefore there exists a function $u \in L^{p}(\Omega \times \Delta(A))$ such that

$$
L(\widehat{f})=\int_{\Omega} \int_{\Delta(A)} u \widehat{f} d \beta d x \quad\left(f \in L^{p^{\prime}}(\Omega ; A)\right) .
$$

This completes the proof.

The notion of $W^{1, p}$-proper $H$-algebras will be of fundamental interest in the ensuing sections.

Definition 2.2. The $H$-algebra $A$ is said to be $W^{1, p}$-proper for some given real $p>1$ if the following conditions are fulfilled:

$(\mathrm{PR})_{1} \mathcal{D}(\Delta(A))$ is dense in $W^{1, p}(\Delta(A))$;

$(\mathrm{PR})_{2}$ For any bounded open set $\Omega$ in $\mathbb{R}_{x}^{N}, W^{1, p}(\Omega)$ is $\Sigma$-reflexive in the following sense: given a fundamental sequence $E$ and a sequence $\left(u_{\varepsilon}\right)_{\varepsilon \in E}$ which is bounded in $W^{1, p}(\Omega)$, one can extract a subsequence $E^{\prime}$ from $E$ such that as $E^{\prime} \ni \varepsilon \rightarrow 0$, we have $u_{\varepsilon} \rightarrow u_{0}$ in $W^{1, p}(\Omega)$-weak and $\frac{\partial u_{\varepsilon}}{\partial x_{i}} \rightarrow \frac{\partial u_{0}}{\partial x_{i}}+\partial_{i} u_{1}$ in $L^{p}(\Omega)$-weak $\Sigma(1 \leq i \leq N)$, where $u_{0} \in W^{1, p}(\Omega)$ and $u_{1} \in L^{p}\left(\Omega ; W_{\#}^{1, p}(\Delta(A))\right)$.

We give here below a few examples of $W^{1, p}$-proper $H$-algebras.

Example 2.1. Let $A=\mathcal{C}_{\text {per }}(Y)\left(Y=(0,1)^{N}\right)$ be the periodic $H$-algebra of $Y$-periodic continuous functions on $\mathbb{R}^{N}$. Then $A$ is $W^{1, p}$-proper for any real number $p>1$ see [21].

Example 2.2. Let $\mathcal{R}$ be a countable subgroup of $\mathbb{R}^{N}$. Let $A=A P_{\mathcal{R}}\left(\mathbb{R}^{N}\right)$ be the algebra of complex almost periodic continuous functions defined earlier in this subsection. Then following the same line of reasoning as in [9]) we see that the $H$-algebra $A$ is $W^{1, p}$-proper for any real number $p>1$.

Example 2.3. Let $\mathcal{R}$ be as in Example 2.2. One define the $H$-algebra of perturbed almost periodic functions as the direct sum $A=A P_{\mathcal{R}}\left(\mathbb{R}^{N}\right) \oplus \mathcal{C}_{0}\left(\mathbb{R}^{N}\right)$ where $\mathcal{C}_{0}\left(\mathbb{R}^{N}\right)$ denotes the space of continuous functions in $\mathbb{R}^{N}$ that vanish at infinity. Then it can be shown using [21] and Example 2.2 that $A$ is a $W^{1, p_{-}}$ proper $H$-algebra for any real number $p>1$. Indeed $A$ is the space denoted by $\mathcal{B}_{\infty, \mathcal{R}}\left(\mathbb{R}^{N}\right)$ defined in [18] as the closure in $\mathcal{B}\left(\mathbb{R}^{N}\right)$ of the space of all finite sums

$$
\sum_{\text {finite }} \varphi_{i} u_{i} \quad\left(\varphi_{i} \in \mathcal{B}_{\infty}\left(\mathbb{R}^{N}\right), u_{i} \in A P_{\mathcal{R}}\left(\mathbb{R}^{N}\right)\right)
$$

where $\mathcal{B}_{\infty}\left(\mathbb{R}^{N}\right)$ is the space of all continuous complex functions on $\mathbb{R}^{N}$ that have finite limit at infinity. In particular for $\mathcal{R}=\{0\}$ we have that $A=$ $\mathbb{R} \oplus \mathcal{C}_{0}\left(\mathbb{R}^{N}\right)=\mathcal{B}_{\infty}\left(\mathbb{R}^{N}\right)$ is $W^{1, p}$-proper for any real number $p>1$. 


\subsection{The abstract problem and preliminary results}

Let $1 \leq p<\infty$, and let $Y=\left(-\frac{1}{2}, \frac{1}{2}\right)^{N}$ be the unit cell. We denote by $\left(L^{p}, \ell^{\infty}\right)\left(\mathbb{R}^{N}\right)[14]$ the space of all $u \in L_{\text {loc }}^{p}\left(\mathbb{R}^{N}\right)$ such that

$$
\|u\|_{p, \infty}=\sup _{k \in \mathbb{Z}^{N}}\left(\int_{k+Y}|u(y)|^{p} d y\right)^{\frac{1}{p}}<+\infty .
$$

This is a Banach space under the norm $\|\cdot\|_{p, \infty}$.

Now, let $A$ be an $H$-algebra on $\mathbb{R}^{N}$ which is assumed to be translation invariant. We define $\mathcal{B}_{A}^{p}\left(\mathbb{R}^{N}\right)$ to be the closure of $A$ in $\left(L^{p}, \ell^{\infty}\right)\left(\mathbb{R}^{N}\right)$. Provided with the norm $\|\cdot\|_{p, \infty}, \mathcal{B}_{A}^{p}\left(\mathbb{R}^{N}\right)$ is a Banach space. It is a generalized Stepanov type space. Since its properties are very closed to those of the generalized Besicovitch type spaces $[8,9]$ we refer the reader to $[8,9]$ for the documented presentation of these spaces. Moreover Propositions 2.3-2.4 and Corollaries 2.1-2.2 of [18] are still valid in the present context. In particular we have the following fundamental results:

1) The mean value $M$ as defined on $A$, extends by continuity to a positive continuous linear form (still denoted by $M$ ) on $\mathcal{B}_{A}^{p}\left(\mathbb{R}^{N}\right)$. Furthermore, for each $u \in \mathcal{B}_{A}^{p}\left(\mathbb{R}^{N}\right)$, we have $u^{\varepsilon} \rightarrow M(u)$ in $L^{p}(\Omega)$-weak as $\varepsilon \rightarrow 0$, where $u^{\varepsilon} \in L^{p}(\Omega)$ is defined by $u^{\varepsilon}(x)=u(x / \varepsilon)$ for $x \in \Omega$;

2) If $1 \leq p, q, r<\infty$ are such that $\frac{1}{p}+\frac{1}{q}=\frac{1}{r}$ and if $u \in \mathcal{B}_{A}^{p}\left(\mathbb{R}^{N}\right)$ and $v \in \mathcal{B}_{A}^{q}\left(\mathbb{R}^{N}\right)$, then $u v \in \mathcal{B}_{A}^{r}\left(\mathbb{R}^{N}\right)$;

3) Property (2.1) (in Definition 2.1) still holds for $\psi \in \mathcal{C}\left(\bar{\Omega} ; \mathcal{B}_{A}^{p^{\prime}, \infty}\right.$ ) where $\mathcal{B}_{A}^{p^{\prime}, \infty}=\mathcal{B}_{A}^{p^{\prime}}\left(\mathbb{R}^{N}\right) \cap L^{\infty}\left(\mathbb{R}^{N}\right)$ and $p^{\prime}=p /(p-1)$. Furthermore, if we provide the space $\mathcal{B}_{A}^{p^{\prime}, \infty}$ with the $L^{\infty}\left(\mathbb{R}^{N}\right)$-norm, it can be shown that, for $u \in \mathcal{B}_{A}^{p^{\prime}, \infty}$, we have $\mathcal{G}(u) \in L^{\infty}(\Delta(A))$ and $\|\mathcal{G}(u)\|_{L^{\infty}(\Delta(A))} \leq\|u\|_{L^{\infty}\left(\mathbb{R}^{N}\right)}$.

4) The Gelfand transformation $\mathcal{G}: A \rightarrow \mathcal{C}(\Delta(A))$ extends by continuity to a unique continuous linear mapping, still denoted by $\mathcal{G}$, of $\mathcal{B}_{A}^{p}\left(\mathbb{R}^{N}\right)$ into $L^{p}(\Delta(A))$.

This being so, the main purpose of this section is to investigate the asymptotic analysis, as $\varepsilon \rightarrow 0$, of $u_{\varepsilon}$ (see (1.6)) under the abstract structure hypothesis

$$
f(x, \cdot, \lambda) \in \mathcal{B}_{A}^{1}\left(\mathbb{R}^{N}\right) \text { for any } x \in \bar{\Omega} \text { and all } \lambda \in \mathbb{R}^{n N} .
$$

where $p^{\prime}=p /(p-1)$ with $1<p<\infty$.

Now, let $A_{\mathbb{R}}=A \cap \mathcal{C}\left(\mathbb{R}_{y}^{N} ; \mathbb{R}\right)$ and $A_{\mathbb{R}}^{\infty}=A^{\infty} \cap A_{\mathbb{R}}$. The following result will be fundamental in the homogenization process.

Proposition 2.3. Assume (2.2) holds true. Then, for every $\Psi \in\left(A_{\mathbb{R}}\right)^{n N}$ and every $x \in \bar{\Omega}$, the function $y \mapsto f(x, y, \Psi(y))$ denoted below by $f(x, \cdot, \Psi)$, lies in $\mathcal{B}_{A}^{1}\left(\mathbb{R}^{N}\right)$.

Proof. Let $K \subset \mathbb{R}^{n N}$ be a compact set such that $\Psi(y) \in K$ for all $y \in \mathbb{R}^{N}$. By viewing $f$ as a function $(x, \lambda) \mapsto f(x, \cdot, \lambda)$ of $\bar{\Omega} \times \mathbb{R}^{n N}$ into $\mathcal{B}_{A}^{1}\left(\mathbb{R}^{N}\right)$, we have that $f$ belongs to $\mathcal{C}\left(\bar{\Omega} \times \mathbb{R}^{n N} ; \mathcal{B}_{A}^{1}\left(\mathbb{R}^{N}\right)\right.$ ) (combine (2.2) with (1.2) and 
(1.4)). Still denoting by $f$ the restriction of this function to $\bar{\Omega} \times K$, it immediately follows that $f \in \mathcal{C}\left(\bar{\Omega} \times K ; \mathcal{B}_{A}^{1}\left(\mathbb{R}^{N}\right)\right)$. Hence using the density of $\mathcal{C}(\bar{\Omega} \times$ $K) \otimes \mathcal{B}_{A}^{1}\left(\mathbb{R}^{N}\right)$ in $\mathcal{C}\left(\bar{\Omega} \times K ; \mathcal{B}_{A}^{1}\left(\mathbb{R}^{N}\right)\right)$, one may consider a sequence $\left(q_{n}\right)_{n \geq 1}$ in $\mathcal{C}(\bar{\Omega} \times K) \otimes \mathcal{B}_{A}^{1}\left(\mathbb{R}^{N}\right)$ such that

$$
\sup _{x \in \bar{\Omega}} \sup _{\lambda \in K}\left\|q_{n}(x, \cdot, \lambda)-f(x, \cdot, \lambda)\right\|_{1, \infty} \rightarrow 0 \quad \text { as } n \rightarrow \infty .
$$

As

$$
\left\|q_{n}(x, \cdot, \Psi)-f(x, \cdot, \Psi)\right\|_{1, \infty} \leq \sup _{x \in \bar{\Omega}} \sup _{\lambda \in K}\left\|q_{n}(x, \cdot, \lambda)-f(x, \cdot, \lambda)\right\|_{1, \infty}
$$

we have $q_{n}(x, \cdot, \Psi) \rightarrow f(x, \cdot, \Psi)$ in $\mathcal{B}_{A}^{1}\left(\mathbb{R}^{N}\right)$ as $n \rightarrow \infty$. Thus, the proposition is shown if we can verify that each $q_{n}(x, \cdot, \Psi)$ lies in $\mathcal{B}_{A}^{1}\left(\mathbb{R}^{N}\right)$. However this will follow in an obvious way once we have checked that for any function $q: \bar{\Omega} \times \mathbb{R}_{y}^{N} \times \mathbb{R}_{\lambda}^{n N} \rightarrow \mathbb{R}$ of the form

$$
\begin{aligned}
& q(x, y, \lambda)=\chi(x, \lambda) \Phi(y)\left(y \in \mathbb{R}^{N}, \lambda \in \mathbb{R}^{n N}, x \in \bar{\Omega}\right) \text { with } \chi \in \mathcal{C}(\bar{\Omega} \times K) \\
& \quad \text { and } \Phi \in \mathcal{B}_{A}^{1}\left(\mathbb{R}^{N}\right),
\end{aligned}
$$

we have $q(x, \cdot, \Psi) \in \mathcal{B}_{A}^{1}\left(\mathbb{R}^{N}\right)$. But given $q$ as above, we know by the StoneWeierstrass theorem that there is a sequence $\left(f_{n}\right)_{n \geq 1}$ of polynomials in $(x, \lambda) \in$ $\bar{\Omega} \times K$ such that $f_{n} \rightarrow \chi$ in $\mathcal{C}(\bar{\Omega} \times K)$ as $n \rightarrow \infty$, hence $f_{n}(x, \Psi) \rightarrow \chi(x, \Psi)$ in $\mathcal{B}\left(\mathbb{R}_{y}^{N}\right)$ as $n \rightarrow \infty$. Therefore, it follows that $\chi(x, \Psi)$ lies in $A_{\mathbb{R}}$, since the same is true for each $f_{n}(x, \Psi)$ (recall that $A$ is an algebra). We conclude that $q(x, \cdot, \Psi)=\chi(x, \Psi) \Phi \in \mathcal{B}_{A}^{1}\left(\mathbb{R}^{N}\right)$ as the product of an element of $A$ by an element of $\mathcal{B}_{A}^{1}\left(\mathbb{R}^{N}\right)$. This concludes the proof.

Now, let $\Psi \in \mathcal{C}\left(\bar{\Omega} ;\left(A_{\mathbb{R}}\right)^{n N}\right)$. It is worth recalling that using inequalities (1.2) and (1.4), one can easily define the function $y \mapsto f(x, y, \Psi(x, y))$ of $\mathbb{R}^{N}$ into $\mathbb{R}_{+}$, denoted by $f(x, \cdot, \Psi(x, \cdot))$, which lies in $L^{\infty}\left(\mathbb{R}_{y}^{N}\right)$, and hence the function $x \mapsto f(x, \cdot, \Psi(x, \cdot))$ of $\bar{\Omega}$ into $L^{\infty}\left(\mathbb{R}_{y}^{N}\right)$ (denoted by $f(\cdot, \cdot, \Psi)$ ) as element of $\mathcal{C}\left(\bar{\Omega} ; L^{\infty}\left(\mathbb{R}_{y}^{N}\right)\right)$. Therefore, for fixed $\varepsilon>0$, we define the function $x \mapsto f\left(x, x / \varepsilon, \Psi(x, x / \varepsilon)\right.$ ) of $\Omega$ into $\mathbb{R}_{+}$(denoted by $f^{\varepsilon}\left(\cdot, \cdot, \Psi^{\varepsilon}\right)$ ) as element of $L^{\infty}(\Omega ; \mathbb{R})$. This function will be of particular interest in the rest of this work. Moreover, in view of (1.2), (1.4) and (2.2), we have

$$
f(\cdot, \cdot, \Psi) \in \mathcal{C}\left(\bar{\Omega} ; \mathcal{B}_{A}^{1}\left(\mathbb{R}^{N}\right)\right) \text { for all } \Psi \in \mathcal{C}\left(\bar{\Omega} ;\left(A_{\mathbb{R}}\right)^{n N}\right) .
$$

Before going any further, however, we require a few preliminary notions and results. To this end, let $1<p<\infty$. Set

$$
\mathbb{F}_{0}^{1, p}=W_{0}^{1, p}\left(\Omega ; \mathbb{R}^{n}\right) \times L^{p}\left(\Omega ; W_{\#}^{1, p}(\Delta(A) ; \mathbb{R})^{n}\right),
$$

where $W_{\#}^{1, p}(\Delta(A) ; \mathbb{R})=\left\{u \in W_{\#}^{1, p}(\Delta(A)): \partial_{i} u \in L^{p}(\Delta(A) ; \mathbb{R})(1 \leq i \leq N)\right\}$ is provided with the $W_{\#}^{1, p}(\Delta(A))$-norm (which makes it a Banach space). We equip $\mathbb{F}_{0}^{1, p}$ with the norm

$$
\|\mathbf{u}\|_{\mathbb{F}_{0}^{1, p}}=\left(\left\|u_{0}\right\|_{W_{0}^{1, p}(\Omega)^{n}}^{p}+\left\|u_{1}\right\|_{L^{p}\left(\Omega ; W_{\#}^{1, p}(\Delta(A))^{n}\right)}^{p}\right)^{\frac{1}{p}} \quad\left(\mathbf{u}=\left(u_{0}, u_{1}\right) \in \mathbb{F}_{0}^{1, p}\right)
$$


where $\|\cdot\|_{W_{0}^{1, p}(\Omega)^{n}}$ is defined in Sect. 1 , and $\left\|u_{1}\right\|_{L^{p}\left(\Omega ; W_{\#}^{1, p}(\Delta(A))^{n}\right)}=\left(\sum_{i=1}^{n} \sum_{j=1}^{N}\left\|\partial_{j} u_{1, i}\right\|_{L^{p}(\Omega \times \Delta(A))}^{p}\right)^{\frac{1}{p}}$ for $u_{1}=\left(u_{1, i}\right)_{1 \leq i \leq n}$. With this norm, $\mathbb{F}_{0}^{1, p}$ is a Banach space. In the sequel we assume that $\mathcal{D}(\Delta(A))$ is dense in $W^{1, p}(\Delta(A))$. Then $F_{0}^{\infty}=\mathcal{D}(\Omega ; \mathbb{R})^{n} \times\left[\mathcal{D}(\Omega ; \mathbb{R}) \otimes J(\mathcal{D}(\Delta(A) ; \mathbb{R}) / \mathbb{C})^{n}\right]$ is dense in $\mathbb{F}_{0}^{1, p}$, where $\mathcal{D}(\Delta(A) ; \mathbb{R}) / \mathbb{C}=\left\{u \in \mathcal{D}(\Delta(A) ; \mathbb{R}): \int_{\Delta(A)} u d \beta=0\right\}$.

In all that follows, we assume that $(2.2)$ holds. Then $f(x, \cdot, \Psi) \in \mathcal{B}_{A}^{1, \infty}$ $\left(\mathbb{R}^{N}\right)=\mathcal{B}_{A}^{1}\left(\mathbb{R}^{N}\right) \cap L^{\infty}\left(\mathbb{R}^{N} ; \mathbb{R}\right)$ for all $x \in \bar{\Omega}$ and for all $\Psi \in\left(A_{\mathbb{R}}\right)^{n N}$. Consequently $\mathcal{G}(f(x, \cdot, \Psi)) \in L^{\infty}(\Delta(A))$ for all $x \in \bar{\Omega}$ (see [18, Corollary 2.2]). This being so, for $\varphi \in \mathcal{C}(\Delta(A) ; \mathbb{R})^{n N}$ and $x \in \bar{\Omega}$, let

$$
b(x, \varphi)=\mathcal{G}\left(f\left(x, \cdot, \mathcal{G}^{-1} \varphi\right)\right)
$$

where $\mathcal{G}^{-1} \varphi=\left(\mathcal{G}^{-1} \varphi_{j}\right)_{1 \leq j \leq n N}$. Then, for fixed $x \in \bar{\Omega}$, one defines a transformation $b(x, \cdot)$ from $\mathcal{C}(\Delta(A) ; \mathbb{R})^{n N}$ into $L^{\infty}(\Delta(A))$.

The following result will allow us to rigorously set the homogenized problem.

Proposition 2.4. Suppose that (2.2) holds. For $\Psi \in \mathcal{C}\left(\bar{\Omega} ;\left(A_{\mathbb{R}}\right)^{n N}\right)$, let $b(x$, $\widehat{\Psi}(x))=\mathcal{G}(f(x, \cdot, \Psi(x, \cdot)))(x \in \bar{\Omega})$ where $\widehat{\Psi}=\left(\mathcal{G} \circ \psi_{j}\right)_{1 \leq j \leq n N}$ with $\Psi=$ $\left(\psi_{j}\right)_{1 \leq j \leq n N}$. This defines a mapping $x \mapsto b(x, \widehat{\Psi}(x))$, denoted by $b(\cdot, \widehat{\Psi})$, of $\bar{\Omega}$ into $L^{\infty}(\Delta(A))$. Furthermore, the following assertions are true:

(i) $b(\cdot, \widehat{\Psi}) \in \mathcal{C}\left(\bar{\Omega} ; L^{\infty}(\Delta(A))\right)$ and

$$
f^{\varepsilon}\left(\cdot, \cdot, \Psi^{\varepsilon}\right) \rightarrow b(\cdot, \widehat{\Psi}) \text { in } L^{1}(\Omega) \text {-weak } \Sigma \text { as } \varepsilon \rightarrow 0 .
$$

(ii) The mapping $\Phi \mapsto b(\cdot, \Phi)$ of $\mathcal{C}\left(\bar{\Omega} ; \mathcal{C}(\Delta(A) ; \mathbb{R})^{n N}\right)$ into $L^{1}(\Omega \times \Delta(A))$ extends by continuity to a mapping, denoted by b, of $L^{p}(\Omega \times \Delta(A) ; \mathbb{R})^{n N}$ into $L^{1}(\Omega \times \Delta(A) ; \mathbb{R})$ such that

$$
\begin{aligned}
& \|b(\cdot, \mathbf{u})-b(\cdot, \mathbf{v})\|_{L^{1}(\Omega \times \Delta(A))} \\
& \quad \leq c\left(1+\|\mathbf{u}\|_{L^{p}(\Omega \times \Delta(A))^{n N}}^{p-1}+\|\mathbf{v}\|_{L^{p}(\Omega \times \Delta(A))^{n N}}^{p-1}\right)^{\frac{p-1}{p}}\|\mathbf{u}-\mathbf{v}\|_{L^{p}(\Omega \times \Delta(A))^{n N}} \\
& \quad \text { for all } \mathbf{u}, \mathbf{v} \in L^{p}(\Omega \times \Delta(A) ; \mathbb{R})^{n N} .
\end{aligned}
$$

Proof. Let $\Psi \in \mathcal{C}\left(\bar{\Omega} ;\left(A_{\mathbb{R}}\right)^{n N}\right)$. Thanks to the preceding proposition, assuming (2.2) holds, then the function $(x, y) \mapsto f(x, y, \Psi(x, y))$ is defined as an element of $\mathcal{C}\left(\bar{\Omega} ; \mathcal{B}_{A}^{1, \infty}\right)$ denoted by $f(\cdot, \cdot, \Psi)$ or explicitly by $f(x, y, \Psi)$. Now, since property (2.1) (in Definition 2.1) still holds for $v \in \mathcal{C}\left(\bar{\Omega} ; \mathcal{B}_{A}^{1, \infty}\right)$ and thanks to the result 1) stated previously, the convergence result (2.3) follows at once. On the other hand, by the definition of the function $f(\cdot, \cdot, \Psi)$ it is immediate that this function verifies property of the same type as in (1.4), so that arguing as in the proof of [22, Proposition 3.1] we get the remainder of the above proposition, that is, (ii). 
Remark 2.2. We have in particular

(i) $\quad c_{1}|\lambda|^{p} \leq b(x, \lambda) \leq c_{2}\left(1+|\lambda|^{p}\right) \quad\left(x \in \Omega, \lambda \in \mathbb{R}^{n N}\right)$;

(ii) $b(x, \cdot)$ is strictly convex for all $x \in \Omega$;

(iii) $b(\cdot, \lambda)$ is measurable for all $\lambda \in \mathbb{R}^{n N}$.

The next corollary will be of great interest in the proof of the main result of the paper.

Corollary 2.5. Let

$$
\Phi_{\varepsilon}=\psi_{0}+\varepsilon \psi_{1}^{\varepsilon},
$$

i.e., $\Phi_{\varepsilon}(x)=\psi_{0}(x)+\varepsilon \psi_{1}(x, x / \varepsilon)$ for $x \in \Omega$, where $\psi_{0} \in \mathcal{D}(\Omega ; \mathbb{R})^{n}$ and $\psi_{1} \in$ $\left(\mathcal{D}(\Omega ; \mathbb{R}) \otimes A_{\mathbb{R}}^{\infty}\right)^{n}$. Then, as $\varepsilon \rightarrow 0$,

$$
f^{\varepsilon}\left(\cdot, \cdot D \Phi_{\varepsilon}\right) \rightarrow b\left(\cdot, D \psi_{0}+\partial \widehat{\psi}_{1}\right) \text { in } L^{1}(\Omega)-\text { weak } \Sigma
$$

where $\partial \widehat{\psi}_{1}=\left(\partial_{j} \widehat{\psi}_{1}\right)_{1 \leq j \leq N}$.

Proof. Since $D \Phi_{\varepsilon}=D \psi_{0}+\left(D_{y} \psi_{1}\right)^{\varepsilon}+\varepsilon\left(D \psi_{1}\right)^{\varepsilon}$, it is immediate by (1.4) (where we have taken there $y=x / \varepsilon, \lambda=D \psi_{0}+\left(D_{y} \psi_{1}\right)^{\varepsilon}$ and $\lambda^{\prime}=D \Phi_{\varepsilon}$, and after integrating the resultant inequality over $\Omega$ ) that, as $\varepsilon \rightarrow 0$,

$$
f^{\varepsilon}\left(\cdot, \cdot, D \Phi_{\varepsilon}\right)-f^{\varepsilon}\left(\cdot, \cdot, D \psi_{0}+\left(D_{y} \psi_{1}\right)^{\varepsilon}\right) \rightarrow 0 \text { in } L^{1}(\Omega) .
$$

Thus by the decomposition (for $v \in L^{\infty}(\Omega ; A)$ )

$$
\begin{aligned}
\int_{\Omega} f^{\varepsilon}\left(\cdot, \cdot, D \Phi_{\varepsilon}\right) v^{\varepsilon} d x-\iint_{\Omega \times \Delta(A)} b\left(\cdot, D \psi_{0}+\partial \widehat{\psi_{1}}\right) \widehat{v} d x d \beta \\
=\int_{\Omega}\left[f^{\varepsilon}\left(\cdot, \cdot, D \Phi_{\varepsilon}\right)-f^{\varepsilon}\left(\cdot, \cdot, D \psi_{0}+\left(D_{y} \psi_{1}\right)^{\varepsilon}\right)\right] v^{\varepsilon} d x \\
\quad+\int_{\Omega} f^{\varepsilon}\left(\cdot, \cdot, D \psi_{0}+\left(D_{y} \psi_{1}\right)^{\varepsilon}\right) v^{\varepsilon} d x \\
\quad-\iint_{\Omega \times \Delta(A)} b\left(\cdot, D \psi_{0}+\partial \widehat{\psi}_{1}\right) \widehat{v} d x d \beta
\end{aligned}
$$

the result follows at once by (2.6) and by [part (i) of] Proposition 2.3.

Now, for $\mathbf{v}=\left(v_{0}, v_{1}\right) \in \mathbb{F}_{0}^{1, p}$, set $\mathbb{D} \mathbf{v}=D v_{0}+\partial v_{1} \in L^{p}(\Omega \times \Delta(A))^{n N}$ and define the functional $F$ on $\mathbb{F}_{0}^{1, p}$ by

$$
F(\mathbf{v})=\iint_{\Omega \times \Delta(A)} b(\cdot, \mathbb{D} \mathbf{v}) d x d \beta .
$$

Thanks to Remark 2.2, there exists a unique function $\mathbf{u}=\left(u_{0}, u_{1}\right) \in \mathbb{F}_{0}^{1, p}$ that minimizes $F$ on $\mathbb{F}_{0}^{1, p}$, i.e.,

$$
F(\mathbf{u})=\inf _{\mathbf{v} \in \mathbb{F}_{0}^{1, p}} F(\mathbf{v}) .
$$




\subsection{Regularization and partial results}

Let $\theta_{m} \in \mathcal{D}\left(\mathbb{R}^{n N}\right)$ (integer $m \geq 1$ ) with $0 \leq \theta_{m}, \operatorname{supp} \theta_{m} \subset \frac{1}{m} \bar{B}_{n N}\left(B_{n N}\right.$ the open unit ball in $\mathbb{R}^{n N}, \bar{B}_{n N}$ its closure in $\mathbb{R}^{n N}$ ) and $\int \theta_{m}(\lambda) d \lambda=1$. We regularize the integrand $f$ in $F_{\varepsilon}$ in order to get an approximating family of integrands $\left(f_{m}\right)_{m \geq 1}$ satisfying standard growth conditions of order $p$ and differentiability as follows:

$$
f_{m}(x, y, \lambda)=\int \theta_{m}(\eta) f(x, y, \lambda-\eta) d \eta\left((x, y, \lambda) \in \mathbb{R}^{N} \times \mathbb{R}^{N} \times \mathbb{R}^{n N}\right) .
$$

The main properties of this new integrand are the following:

$\left(\mathrm{H}_{1}\right)_{m}$ We have

$$
\left|f_{m}(x, y, \lambda)-f_{m}\left(x^{\prime}, y, \lambda\right)\right| \leq \omega\left(\left|x-x^{\prime}\right|\right)\left(a(y)+f_{m}(x, y, \lambda)\right)
$$

for all $x, x^{\prime} \in \mathbb{R}^{N}, \lambda \in \mathbb{R}^{n N}$ and for almost all $y \in \mathbb{R}^{N}$, where the functions $\omega$ and $a$ are as in $\left(\mathrm{H}_{1}\right)$ (see Sect. 1),

$\left(\mathrm{H}_{2}\right)_{m} f_{m}(x, \cdot, \lambda)$ is measurable for all $(x, \lambda) \in \mathbb{R}^{N} \times \mathbb{R}^{n N}$,

$\left(\mathrm{H}_{3}\right)_{m} f_{m}(x, y, \cdot)$ is convex for almost all $y \in \mathbb{R}^{N}$ and for all $x \in \mathbb{R}^{N}$,

$\left(\mathrm{H}_{4}\right)_{m}$ There exist $0<c_{3} \leq c_{4}, c_{3}$ depending or not to $m$ and $c_{4}$ independent to $m$, such that

$c_{3}|\lambda|^{p} \leq f_{m}(x, y, \lambda) \leq c_{4}\left(1+|\lambda|^{p}\right)\left(x \in \mathbb{R}^{N}, \lambda \in \mathbb{R}^{n N}\right.$, a.e. $\left.y \in \mathbb{R}^{N}\right)(m \geq 1)$

$\left(\mathrm{H}_{5}\right)_{m} \frac{\partial f_{m}}{\partial \lambda}(x, y, \lambda)$ exists for all $(x, \lambda)$ and for almost all $y \in \mathbb{R}^{N}$, and there exists a constant $c>0$ depending on $m$ such that

$$
\left|\frac{\partial f_{m}}{\partial \lambda}(x, y, \lambda)\right| \leq c\left(1+|\lambda|^{p-1}\right)
$$

for all $(x, \lambda) \in \mathbb{R}^{N} \times \mathbb{R}^{n N}$ and for almost all $y \in \mathbb{R}^{N}(m \geq 1)$.

Properties $\left(\mathrm{H}_{1}\right)_{m^{-}}\left(\mathrm{H}_{5}\right)_{m}$ come easily from properties $\left(\mathrm{H}_{1}\right)-\left(\mathrm{H}_{4}\right)$ and from the Riemann-Lebesgue theorem.

Lemma 2.6. Assume (2.2) holds. Then for any integer $m \geq 1$ and any function $\Psi \in\left(A_{\mathbb{R}}\right)^{n N}$ we have $f_{m}(x, \cdot, \Psi) \in \mathcal{B}_{A}^{1}$ for all $x \in \bar{\Omega}$.

The proof of the preceding lemma strongly relies on the following proposition whose proof can be found in [4].

Proposition 2.7 ([4, Chap. III, Sect. 3, no 3, Proposition 7]). Let $F$ be a locally convex topological vector space which is separated, and let $X$ be a locally compact topological space provided with some Radon measure $\mu$. Finally let $f$ : $X \rightarrow F$ be a continuous function with compact support. If the range $f(X)$ of $f$ is contained in a complete convex subset of $F$, then the integral of $f, \int f d \mu$, lies in $F$.

We can now prove the previous lemma.

Proof of Lemma 2.6. Fix an integer $m \geq 1$. Since the function $f_{m}$ satisfies properties $\left(\mathrm{H}_{1}\right)_{m}-\left(\mathrm{H}_{4}\right)_{m}$, as a result of Proposition 2.3 we only need to check the following:

$$
f_{m}(x, \cdot, \lambda) \in \mathcal{B}_{A}^{1} \quad \text { for all }(x, \lambda) \in \bar{\Omega} \times \mathbb{R}^{n N} .
$$


For that purpose, set $g(y, \eta)=\theta_{m}(\eta) f(x, y, \lambda-\eta)\left(y \in \mathbb{R}^{N}, \eta \in \mathbb{R}^{n N}\right)$ for $x$ and $\lambda$ arbitrarily fixed. Then by $(2.2)$ one has $g(\cdot, \eta) \in \mathcal{B}_{A}^{1}$ for any $\eta \in \mathbb{R}^{n N}$, and moreover combining (1.4) with the fact that $\theta_{m} \in \mathcal{D}\left(\mathbb{R}^{n N}\right)$ we easily deduce that the function $\eta \mapsto g(\cdot, \eta)$, is an element of $\mathcal{K}\left(\mathbb{R}^{n N} ; \mathcal{B}_{A}^{1}\right)$ (the space of continuous functions of $\mathbb{R}^{n N}$ into $\mathcal{B}_{A}^{1}$ with compact support). Thus, by virtue of Proposition 2.7 above, we get $\int g(\cdot, \eta) d \eta \in \mathcal{B}_{A}^{1}$, i.e., (2.8).

Now, fix $m \geq 1$. We know by the preceding lemma that $f_{m}(x, \cdot, \Psi) \in \mathcal{B}_{A}^{1}$ for all $\Psi \in\left(A_{\mathbb{R}}\right)^{n N}$ and for all $x \in \bar{\Omega}$. Hence by setting

$$
b_{m}(x, \widehat{\Psi}(x))=\mathcal{G}\left(f_{m}(x, \cdot, \Psi(x, \cdot))\right) \text { for } \Psi \in \mathcal{C}\left(\bar{\Omega} ;\left(A_{\mathbb{R}}\right)^{n N}\right) \text { and } x \in \bar{\Omega},
$$

we have the results similar to those in Proposition 2.4 mutatis mutandis. Moreover the following result holds.

Lemma 2.8. Let $u \in L^{p}(\Omega \times \Delta(A) ; \mathbb{R})^{n N}$. Then, as $m \rightarrow \infty$,

$$
b_{m}(\cdot, u) \rightarrow b(\cdot, u) \text { in } L^{1}(\Omega \times \Delta(A)) .
$$

Proof. This will be done in two steps.

Step 1. Let $\Psi \in \mathcal{C}\left(\bar{\Omega} ;\left(A_{\mathbb{R}}\right)^{n N}\right)$; then

$$
\begin{aligned}
& \left\|b_{m}(\cdot, \widehat{\Psi})-b(\cdot, \widehat{\Psi})\right\|_{L^{1}(\Omega \times \Delta(A))}=\iint_{\Omega \times \Delta(A)}\left|b_{m}(x, \widehat{\Psi}(x))-b(x, \widehat{\Psi}(x))\right| d x d \beta \\
& \quad=\iint_{\Omega \times \Delta(A)}\left|\mathcal{G}\left(f_{m}(x, \cdot, \Psi(x, \cdot))-f(x, \cdot, \Psi(x, \cdot))\right)\right| d x d \beta \\
& \quad=\int_{\Omega}\left\|\mathcal{G}\left(f_{m}(x, \cdot, \Psi(x, \cdot))-f(x, \cdot, \Psi(x, \cdot))\right)\right\|_{L^{1}(\Delta(A))} d x \\
& \leq \int_{\Omega}\left\|f_{m}(x, \cdot, \Psi(x, \cdot))-f(x, \cdot, \Psi(x, \cdot))\right\|_{1, \infty} d x \\
& =\int_{\Omega}\left(\sup _{k \in Y} \int_{k+Y}\left|f_{m}(x, y, \Psi(x, y))-f(x, y, \Psi(x, y))\right| d y\right) d x \\
& \leq \int_{\Omega}\left(\sup _{k \in Y} \int_{k+Y}\left|\int \theta_{m}(\eta)[f(x, y, \Psi(x, y)-\eta)-f(x, y, \Psi(x, y))] d \eta\right| d y\right) d x \\
& \leq \frac{c|\Omega|}{m} \sup _{x \in \Omega} \sup _{|\eta| \leq 1 / m} \sup _{z \in \mathbb{R}^{N}}\left(1+|\Psi(x, z)-\eta|^{p-1}+|\Psi(x, z)|^{p-1}\right)
\end{aligned}
$$

where $|\Omega|=\int_{\Omega} d x$. It is easily shown that the right-hand side of the last inequality above goes to zero whenever $m \rightarrow \infty$.

Step 2. Now let $u \in L^{p}(\Omega \times \Delta(A) ; \mathbb{R})^{n N}$; and let $\left(\Psi_{j}\right)$ be a sequence in $\mathcal{C}\left(\bar{\Omega} ;\left(A_{\mathbb{R}}\right)^{n N}\right)$ such that $\widehat{\Psi}_{j} \rightarrow u$ in $L^{p}(\Omega \times \Delta(A))^{n N}$ as $j \rightarrow \infty$. The sequence $\left(\widehat{\Psi}_{j}\right)_{j}$ being bounded in $L^{p}(\Omega \times \Delta(A))^{n N}$, there exists a positive constant $c^{\prime}$ such that

$$
c^{\prime}>2 c \sup _{j \in \mathbb{N}}\left(1+\|u\|_{L^{p}(\Omega \times \Delta(A))^{n N}}^{p-1}+\left\|\widehat{\Psi}_{j}\right\|_{L^{p}(\Omega \times \Delta(A))^{n N}}^{p-1}\right)^{\frac{1}{p^{\prime}}},
$$


where the constant $c$ is as in (2.4). Finally, let $\delta>0$, and let $j_{0} \in \mathbb{N}$ be such that $\left\|u-\widehat{\Psi}_{j_{0}}\right\|_{L^{p}(\Omega \times \Delta(A))^{n N}}<\delta / 2 c^{\prime}$ (remind that $\widehat{\Psi}_{j} \rightarrow u$ in $L^{p}(\Omega \times \Delta(A))^{n N}$ as $j \rightarrow \infty)$. In view of Step 1 there exists an integer $m_{0} \geq 0$ such that

$$
\left\|b_{m}\left(\cdot, \widehat{\Psi}_{j_{0}}\right)-b\left(\cdot, \widehat{\Psi}_{j_{0}}\right)\right\|_{L^{1}(\Omega \times \Delta(A))}<\delta / 2 \text { whenever } m \geq m_{0}
$$

Whence for any $m \geq m_{0}$ large enough, we get $\left\|b_{m}(\cdot, u)-b(\cdot, u)\right\|_{L^{1}(\Omega \times \Delta(A))}<\delta$, where we have used the inequality

$$
\begin{aligned}
& \left\|b_{m}(\cdot, u)-b(\cdot, u)\right\|_{L^{1}(\Omega \times \Delta(A))} \leq\left\|b_{m}(\cdot, u)-b_{m}\left(\cdot, \widehat{\Psi}_{j_{0}}\right)\right\|_{L^{1}(\Omega \times \Delta(A))} \\
& \quad+\left\|b_{m}\left(\cdot, \widehat{\Psi}_{j_{0}}\right)-b\left(\cdot, \widehat{\Psi}_{j_{0}}\right)\right\|_{L^{1}(\Omega \times \Delta(A))}+\left\|b\left(\cdot, \widehat{\Psi}_{j_{0}}\right)-b(\cdot, u)\right\|_{L^{1}(\Omega \times \Delta(A))} \\
& \leq 2 c\left(1+\|u\|_{L^{p}(\Omega \times \Delta(A))^{n N}}^{p-1}+\left\|\widehat{\Psi}_{j_{0}}\right\|_{L^{p}(\Omega \times \Delta(A))^{n N}}^{p-1}\right)^{\frac{1}{p^{\prime}}} \\
& \quad \times\left\|u-\widehat{\Psi}_{j_{0}}\right\|_{L^{p}(\Omega \times \Delta(A))^{n N}}+\left\|b_{m}\left(\cdot, \widehat{\Psi}_{j_{0}}\right)-b\left(\cdot, \widehat{\Psi}_{j_{0}}\right)\right\|_{L^{1}(\Omega \times \Delta(A))}
\end{aligned}
$$

This completes the proof.

Remark 2.3. Note that the way the function $\frac{\partial f_{m}}{\partial \lambda_{i}}$ is defined implies that for $\left.\Psi \in \mathcal{C}\left(\bar{\Omega} ;\left(A_{\mathbb{R}}\right)^{n N}\right)\right)$, the function $x \mapsto \frac{\partial f_{m}}{\partial \lambda_{i}}(x, \cdot, \Psi(x, \cdot))$ from $\bar{\Omega}$ into $L^{\infty}\left(\mathbb{R}_{y}^{N}\right)$ is well defined. We denote this function by $\frac{\partial f_{m}}{\partial \lambda_{i}}(\cdot, \cdot, \Psi)$.

We are now ready to prove one of the most important results of this work.

Proposition 2.9. Let $\left(v_{\varepsilon}\right)_{\varepsilon \in E}$ be a sequence in $L^{p}(\Omega ; \mathbb{R})^{n N}$ which weakly $\Sigma$ converges componentwise to $v \in L^{p}(\Omega \times \Delta(A) ; \mathbb{R})^{n N}$. Assume that $(2.2)$ holds and that for any integer $m$ we have

$$
\frac{\partial f_{m}}{\partial \lambda_{i}}(x, \cdot, \lambda) \in \mathcal{B}_{A}^{p^{\prime}} \text { for any } \lambda \in \mathbb{R}^{n N} \text {, for all } x \in \bar{\Omega} \text { and all } 1 \leq i \leq n N \text {. }
$$

Then for any integer $m \geq 1$ we have

$$
\iint_{\Omega \times \Delta(A)} b_{m}(x, v) d x d \beta \leq \liminf _{E \ni \varepsilon \rightarrow 0} \int_{\Omega} f_{m}\left(x, \frac{x}{\varepsilon}, v_{\varepsilon}(x)\right) d x .
$$

Proof. We follow an argument of Allaire [1]. Let $\Psi \in\left(\mathcal{D}(\Omega ; \mathbb{R}) \otimes A_{\mathbb{R}}\right)^{n N}$ and let $m \geq 1$ be freely fixed. Since $f_{m}(x, y, \cdot)$ is convex and differentiable we have

$$
\begin{aligned}
\int_{\Omega} f_{m}\left(x, \frac{x}{\varepsilon}, v_{\varepsilon}(x)\right) d x \geq & \int_{\Omega} f_{m}\left(x, \frac{x}{\varepsilon}, \Psi\left(x, \frac{x}{\varepsilon}\right)\right) d x \\
& +\int_{\Omega} \frac{\partial f_{m}}{\partial \lambda}\left(x, \frac{x}{\varepsilon}, \Psi\left(x, \frac{x}{\varepsilon}\right)\right) \cdot\left(v_{\varepsilon}(x)-\Psi\left(x, \frac{x}{\varepsilon}\right)\right) d x .
\end{aligned}
$$

In view of Lemma 2.6 we have

$$
\lim _{E \ni \varepsilon \rightarrow 0} \int_{\Omega} f_{m}\left(x, \frac{x}{\varepsilon}, \Psi\left(x, \frac{x}{\varepsilon}\right)\right) d x=\iint_{\Omega \times \Delta(A)} b_{m}(x, \widehat{\Psi}(x)) d x d \beta
$$


where $b_{m}(\cdot, \widehat{\Psi})$ is defined by $(2.9)$. On the other hand, thanks to (2.10) one has $\frac{\partial f_{m}}{\partial \lambda_{i}}(\cdot, \cdot, \Psi) \in \mathcal{C}\left(\bar{\Omega} ; \mathcal{B}_{A}^{p^{\prime}, \infty}\right)$ where $\frac{\partial f_{m}}{\partial \lambda_{i}}(\cdot, \cdot, \Psi)$ stands for the function $x \mapsto$ $\frac{\partial f_{m}}{\partial \lambda_{i}}(x, \cdot, \Psi(x, \cdot))$ from $\bar{\Omega}$ into $\mathcal{B}_{A}^{p^{\prime}}$. Whence, when $E \ni \varepsilon \rightarrow 0$, as a result of [18, Proposition 4.5], one has

$$
\begin{aligned}
& \int_{\Omega} \frac{\partial f_{m}}{\partial \lambda}\left(x, \frac{x}{\varepsilon}, \Psi\left(x, \frac{x}{\varepsilon}\right)\right) \cdot\left(v_{\varepsilon}(x)-\Psi\left(x, \frac{x}{\varepsilon}\right)\right) d x \\
& \rightarrow \iint_{\Omega \times \Delta(A)} \frac{\widehat{\partial f_{m}}}{\partial \lambda}(x, \cdot \Psi(x, \cdot)) \cdot(v(x)-\widehat{\Psi}(x)) d x d \beta
\end{aligned}
$$

where $\frac{\widehat{\partial f_{m}}}{\partial \lambda}(x, \cdot, \Psi(x, \cdot))=\left(\mathcal{G}\left(\frac{\partial f_{m}}{\partial \lambda_{i}}(x, \cdot, \Psi(x, \cdot))\right)\right)_{1 \leq i \leq n N}, \mathcal{G}$ being here the canonical mapping of $\mathcal{B}_{A}^{p^{\prime}}$ into $L^{p^{\prime}}(\Delta(A))$. Therefore

$$
\begin{aligned}
& \liminf _{E \ni \varepsilon \rightarrow 0} \int_{\Omega} f_{m}\left(x, \frac{x}{\varepsilon}, v_{\varepsilon}(x)\right) d x \geq \iint_{\Omega \times \Delta(A)} b_{m}(x, \widehat{\Psi}(x)) d x d \beta \\
& \quad+\iint_{\Omega \times \Delta(A)} \frac{\widehat{\partial f_{m}}}{\partial \lambda}(x, \cdot, \Psi(x, \cdot)) \cdot(v(x)-\widehat{\Psi}(x)) d x d \beta .
\end{aligned}
$$

But, we have to take into account property $\left(\mathrm{H}_{m}\right)_{5}$

$$
\begin{aligned}
& \left|\iint_{\Omega \times \Delta(A)} \frac{\widehat{\partial f_{m}}}{\partial \lambda}(x, \cdot, \Psi(x, \cdot)) \cdot(v(x)-\widehat{\Psi}(x)) d x d \beta\right| \\
& \quad \leq 2^{\frac{1}{p}} c^{\frac{1}{p^{\prime}}}\left[|\Omega|+\|\widehat{\Psi}\|_{L^{p}(\Omega \times \Delta(A))^{n N}}^{p}\right]^{\frac{1}{p^{\prime}}}\|v-\widehat{\Psi}\|_{L^{p}(\Omega \times \Delta(A))^{n N}} .
\end{aligned}
$$

Thus, by choosing a sequence $\left(\Psi_{l}\right)_{l}$ in $\left(\mathcal{D}(\Omega ; \mathbb{R}) \otimes A_{\mathbb{R}}\right)^{n N}$ such that $\widehat{\Psi}_{l} \rightarrow v$ in $L^{p}(\Omega \times \Delta(A))^{n N}$ as $l \rightarrow \infty$, it follows that for fixed $\delta>0$, there exists $l_{0} \in \mathbb{N}$ such that

$$
\left|\iint_{\Omega \times \Delta(A)} \frac{\widehat{\partial f_{m}}}{\partial \lambda}\left(x, \cdot, \Psi_{l}(x, \cdot)\right) \cdot\left(v(x)-\widehat{\Psi}_{l}(x)\right) d x d \beta\right| \leq \delta \quad \text { for all } l \geq l_{0} .
$$

Hence

$$
\begin{aligned}
& \liminf _{E \ni \varepsilon \rightarrow 0} \int_{\Omega} f_{m}\left(x, \frac{x}{\varepsilon}, v_{\varepsilon}(x)\right) d x \\
& \quad \geq \iint_{\Omega \times \Delta(A)} b_{m}\left(x, \widehat{\Psi}_{l}(x)\right) d x d \beta-\delta \quad \text { for all } l \geq l_{0} .
\end{aligned}
$$

Passing to the limit as $l \rightarrow \infty$, and using the continuity of $\lambda \mapsto b_{m}(x, \lambda)$ yield

$$
\liminf _{E \ni \varepsilon \rightarrow 0} \int_{\Omega} f_{m}\left(x, \frac{x}{\varepsilon}, v_{\varepsilon}(x)\right) d x \geq \iint_{\Omega \times \Delta(A)} b_{m}(x, v(x)) d x d \beta-\delta .
$$

Since $\delta$ is arbitrarily fixed, we are led to

$$
\liminf _{E \ni \varepsilon \rightarrow 0} \int_{\Omega} f_{m}\left(x, \frac{x}{\varepsilon}, v_{\varepsilon}(x)\right) d x \geq \iint_{\Omega \times \Delta(A)} b_{m}(x, v(x)) d x d \beta,
$$

which completes the proof. 
Remark 2.4. We shall see in Sect. 4 that hypothesis (2.10) will always be satisfied for the concrete problems that we will consider.

As a consequence of the preceding proposition we have the following

Corollary 2.10. Let $\left(u_{\varepsilon}\right)_{\varepsilon \in E}$ be a sequence in $W^{1, p}\left(\Omega ; \mathbb{R}^{n}\right)$. Assume that $\left(D u_{\varepsilon}\right)_{\varepsilon \in E}$ weakly $\Sigma$-converges componentwise to $\mathbb{D} \mathbf{u}=D u_{0}+\partial u_{1} \in L^{p}(\Omega \times$ $\Delta(A) ; \mathbb{R})^{n N}$, where $\mathbf{u}=\left(u_{0}, u_{1}\right) \in \mathbb{F}_{0}^{1, p}$. Further assume that (2.2) and (2.10) hold. Then

$$
\iint_{\Omega \times \Delta(A)} b(x, \mathbb{D} \mathbf{u}) d x d \beta \leq \liminf _{E \ni \varepsilon \rightarrow 0} \int_{\Omega} f\left(x, \frac{x}{\varepsilon}, D u_{\varepsilon}(x)\right) d x .
$$

Proof. For any $\eta \in \mathbb{R}^{n N}$ one has

$$
f(x, y, \lambda-\eta) \leq f(x, y, \lambda)+c_{2}\left(1+|\lambda-\eta|^{p-1}+|\lambda|^{p-1}\right)|\eta|(\text { see }(1.4)),
$$

hence

$$
f_{m}(x, y, \lambda) \leq f(x, y, \lambda)+c_{2} \int \theta_{m}(\eta)\left(1+|\lambda-\eta|^{p-1}+|\lambda|^{p-1}\right)|\eta| d \eta .
$$

Thus

$$
f_{m}(x, y, \lambda) \leq f(x, y, \lambda)+\frac{c_{2}}{m} \sup _{|\eta| \leq \frac{1}{m}}\left(1+|\lambda-\eta|^{p-1}+|\lambda|^{p-1}\right),
$$

so that

$$
\begin{aligned}
& \int_{\Omega} f_{m}\left(x, \frac{x}{\varepsilon}, D u_{\varepsilon}(x)\right) d x \leq \int_{\Omega} f\left(x, \frac{x}{\varepsilon}, D u_{\varepsilon}(x)\right) d x \\
& +\frac{c_{2}}{m} \int_{\Omega}\left(\sup _{|\eta| \leq \frac{1}{m}}\left(1+\left|D u_{\varepsilon}(x)-\eta\right|^{p-1}+\left|D u_{\varepsilon}(x)\right|^{p-1}\right)\right) d x .
\end{aligned}
$$

Therefore, using Proposition 2.9 we get

$$
\begin{aligned}
& \iint_{\Omega \times \Delta(A)} b_{m}(x, \mathbb{D} \mathbf{u}) d x d \beta \leq \liminf _{E \ni \varepsilon \rightarrow 0} \int_{\Omega} f_{m}\left(x, \frac{x}{\varepsilon}, D u_{\varepsilon}(x)\right) d x \\
& \leq \liminf _{E \ni \varepsilon \rightarrow 0} \int_{\Omega} f\left(x, \frac{x}{\varepsilon}, D u_{\varepsilon}(x)\right) d x \\
& \quad+\frac{c_{2}}{m} \liminf _{E \ni \varepsilon \rightarrow 0} \int_{\Omega}\left(\sup _{|\eta| \leq \frac{1}{m}}\left(1+\left|D u_{\varepsilon}(x)-\eta\right|^{p-1}+\left|D u_{\varepsilon}(x)\right|^{p-1}\right)\right) d x .
\end{aligned}
$$

By letting $m \rightarrow \infty$, using Lemma 2.8 we are led to $(2.11)$.

\section{Abstract homogenization result}

Our main purpose in this section is to prove the following homogenization result. 
Theorem 3.1. Suppose (2.2) and (2.10) hold and further $A$ is $W^{1, p}$-proper $(1<p<\infty)$. For each real $\varepsilon>0$, let $u_{\varepsilon}$ be the unique solution of (1.6). Then, as $\varepsilon \rightarrow 0$,

$$
\begin{aligned}
& u_{\varepsilon} \rightarrow u_{0} \quad \text { in } W^{1, p}(\Omega)^{n} \text {-weak, } \\
& \frac{\partial u_{\varepsilon}}{\partial x_{i}} \rightarrow \frac{\partial u_{0}}{\partial x_{i}}+\partial_{i} u_{1} \quad \text { in } L^{p}(\Omega)^{n} \text {-weak } \Sigma(1 \leq i \leq N),
\end{aligned}
$$

where $\mathbf{u}=\left(u_{0}, u_{1}\right) \in \mathbb{F}_{0}^{1, p}$ is the unique solution of the minimization problem (2.7).

Proof. In view of the growth conditions in $\left(\mathrm{H}_{4}\right)$, the sequence $\left(u_{\varepsilon}\right)_{\varepsilon>0}$ is bounded in $W_{0}^{1, p}\left(\Omega ; \mathbb{R}^{n}\right)$ and so the sequence $\left(f^{\varepsilon}\left(\cdot, \cdot, D u_{\varepsilon}\right)\right)_{\varepsilon>0}$ is bounded in $L^{1}(\Omega)$. Thus, given an arbitrary fundamental sequence $E$, the properness of $A$ and the compactness of the embedding $W_{0}^{1, p}(\Omega ; \mathbb{R}) \rightarrow L^{p}(\Omega ; \mathbb{R})$ guarantee the existence of a subsequence $E^{\prime}$ from $E$ and a couple $\mathbf{u}=\left(u_{0}, u_{1}\right) \in \mathbb{F}_{0}^{1, p}$ such that (3.1)-(3.2) hold when $E^{\prime} \ni \varepsilon \rightarrow 0$. The real sequence $\left(F_{\varepsilon}\left(u_{\varepsilon}\right)\right)_{\varepsilon>0}$ being bounded (since $\left(u_{\varepsilon}\right)_{\varepsilon>0}$ is bounded in $W_{0}^{1, p}\left(\Omega ; \mathbb{R}^{n}\right)$ ), there exists a subsequence from $E^{\prime}$ still denoted by $E^{\prime}$ such that $\lim _{E^{\prime} \ni \varepsilon \rightarrow 0} F_{\varepsilon}\left(u_{\varepsilon}\right)$ exists. It remains to verify that $\mathbf{u}=\left(u_{0}, u_{1}\right)$ solves $(2.7)$. In fact, if $\mathbf{u}$ solves this problem, then thanks to the uniqueness of the solution of $(2.7)$, the whole sequence $\left(u_{\varepsilon}\right)_{\varepsilon>0}$ will verify (3.1) and (3.2) when $\varepsilon \rightarrow 0$. Thus our only concern here is to show that $\mathbf{u}$ solves problem (2.7). To this end, in view of Corollary 2.10, we have

$$
\iint_{\Omega \times \Delta(A)} b(x, \mathbb{D} \mathbf{u}) d x d \beta \leq \lim _{E^{\prime} \ni \varepsilon \rightarrow 0} \int_{\Omega} f\left(x, \frac{x}{\varepsilon}, D u_{\varepsilon}(x)\right) d x .
$$

On the other hand, let us establish an upper bound for $\int_{\Omega} f\left(x, \frac{x}{\varepsilon}, D u_{\varepsilon}(x)\right) d x$. To do that, let $\Phi=\left(\psi_{0}, J^{n}\left(\widehat{\psi}_{1}\right)\right) \in F_{0}^{\infty}$ with $\psi_{0} \in \mathcal{D}(\Omega ; \mathbb{R})^{n}, \psi_{1}=\left(\psi_{1, i}\right)_{1 \leq i \leq n} \in$ $\left[\mathcal{D}(\Omega ; \mathbb{R}) \otimes\left(A_{\mathbb{R}}^{\infty} / \mathbb{C}\right)^{n}\right], J^{n}\left(\widehat{\psi}_{1}\right)=\left(J \circ \widehat{\psi}_{1, i}\right)_{1 \leq i \leq n}$, where $A_{\mathbb{R}}^{\infty} / \mathbb{C}=\left\{\psi \in A_{\mathbb{R}}^{\infty}:\right.$ $M(\psi)=0\}$. Define $\Phi_{\varepsilon}$ as in Corollary 2.5. Since $u_{\varepsilon}$ is the minimizer, one has

$$
\int_{\Omega} f\left(x, \frac{x}{\varepsilon}, D u_{\varepsilon}(x)\right) d x \leq \int_{\Omega} f\left(x, \frac{x}{\varepsilon}, D \Phi_{\varepsilon}(x)\right) d x
$$

Thus, using Corollary 2.5 we get

$$
\lim _{E^{\prime} \ni \varepsilon \rightarrow 0} \int_{\Omega} f\left(x, \frac{x}{\varepsilon}, D u_{\varepsilon}(x)\right) d x \leq \iint_{\Omega \times \Delta(A)} b\left(x, D \psi_{0}+\partial \widehat{\psi}_{1}\right) d x d \beta
$$

for any $\Phi=\left(\psi_{0}, J^{n}\left(\widehat{\psi}_{1}\right)\right) \in F_{0}^{\infty}$, and by density, for all $\Phi$ in $\mathbb{F}_{0}^{1, p}$. From which,

$$
\lim _{E^{\prime} \ni \varepsilon \rightarrow 0} \int_{\Omega} f\left(x, \frac{x}{\varepsilon}, D u_{\varepsilon}(x)\right) d x \leq \inf _{\mathbf{v} \in \mathbb{F}_{0}^{1, p}} \iint_{\Omega \times \Delta(A)} b(x, \mathbb{D} \mathbf{v}) d x d \beta .
$$

Inequalities (3.3) and (3.4) yield

$$
\iint_{\Omega \times \Delta(A)} b(x, \mathbb{D} \mathbf{u}) d x d \beta=\inf _{\mathbf{v} \in \mathbb{F}_{0}^{1, p}} \iint_{\Omega \times \Delta(A)} b(x, \mathbb{D} \mathbf{v}) d x d \beta,
$$

i.e., (2.7). The proof is complete. 


\section{Some concrete homogenization problems for (1.6)}

This section deals with the study of some concrete homogenization problems for (1.6). Thanks to Proposition 2.3, the way of proceeding will be very simple.

\subsection{Problem I (Periodic setting)}

Our goal in this subsection is to study the homogenization of (1.6) under the periodicity hypothesis:

$$
\begin{aligned}
& \text { For any } k \in \mathbb{Z}^{N}, \text { for all } x \in \bar{\Omega} \text { and all } \lambda \in \mathbb{R}^{n N}, \\
& f(x, y+k, \lambda)=f(x, y, \lambda) \text { a.e. in } y \in \mathbb{R}^{N} .
\end{aligned}
$$

We express this by saying that the function $f(x, \cdot, \lambda)$ (for fixed $x, \lambda$ ) is $Y$ periodic, where $Y=(0,1)^{N}$. The appropriated $H$-algebra here is the periodic $H$-algebra $A=\mathcal{C}_{\text {per }}(Y)$ (the space of $Y$-periodic continuous complex functions on $\mathbb{R}_{y}^{N}$ ). We are immediately led to

$$
\begin{aligned}
& f(x, y+k, \Psi(y+k))=f(x, y, \Psi(y))\left(x \in \bar{\Omega}, k \in \mathbb{Z}^{N} \text {, a.e. in } y \in \mathbb{R}^{N}\right) \\
& \quad \text { for any } \Psi \in\left(A_{\mathbb{R}}\right)^{n N} .
\end{aligned}
$$

Whence, thanks to (4.1) and the right inequality in (1.3),

$$
f(x, \cdot, \lambda) \in L_{\text {per }}^{\infty}(Y) \text { for all } \lambda \in \mathbb{R}^{n N} \text { and for all } x \in \bar{\Omega},
$$

so that, since $L_{\text {per }}^{\infty}(Y) \subset L_{\text {per }}^{1}(Y)$,

$$
f(x, \cdot, \lambda) \in L_{\text {per }}^{1}(Y) \text { for all } \lambda \in \mathbb{R}^{n N} \text { and for all } x \in \bar{\Omega},
$$

where $L_{\text {per }}^{1}(Y)$ (resp. $\left.L_{\text {per }}^{\infty}(Y)\right)$ denotes the space of $Y$-periodic functions in $L_{\text {loc }}^{1}\left(\mathbb{R}^{N}\right)$ (resp. $L^{\infty}\left(\mathbb{R}^{N}\right)$ ). But $L_{\text {per }}^{1}(Y)=\mathcal{B}_{A}^{1}$ and the $H$-algebra $\mathcal{C}_{\text {per }}(Y)$ is $W^{1, p}$-proper for any real number $p>1$. Hence the conclusion of Theorem 3.1 holds with $A=\mathcal{C}_{\text {per }}(Y)$, and with $Y$ in place of $\Delta(A), d y$ in place of $d \beta$ and $D_{y}$ in that of $\partial$ (see [18]). Precisely we have the following result.

Theorem 4.1. Let $1<p<\infty$. For each fixed $\varepsilon>0$, let $u_{\varepsilon}$ be the unique solution to (1.6). Then, as $\varepsilon \rightarrow 0$,

$$
u_{\varepsilon} \rightarrow u_{0} \text { in } W_{0}^{1, p}(\Omega)-\text { weak }
$$

and

$$
\frac{\partial u_{\varepsilon}}{\partial x_{i}} \rightarrow \frac{\partial u_{0}}{\partial x_{i}}+\frac{\partial u_{1}}{\partial y_{i}} \text { in } L^{p}(\Omega)-\text { weak } \Sigma(1 \leq i \leq N)
$$

where the vector function $\mathbf{u}=\left(u_{0}, u_{1}\right) \in \mathbb{F}_{0}^{1, p}$ is the unique solution to the variational minimization problem

$$
F(\mathbf{u})=\inf _{\mathbf{v}=\left(v_{0}, v_{1}\right) \in \mathbb{F}_{0}^{1, p}} \iint_{\Omega \times Y} f\left(x, y, D v_{0}+D_{y} v_{1}\right) d x d y
$$

where $\mathbb{F}_{0}^{1, p}=W_{0}^{1, p}\left(\Omega ; \mathbb{R}^{n}\right) \times L^{p}\left(\Omega ; W_{\#}^{1, p}(Y)^{n}\right)$ with $W_{\#}^{1, p}(Y)=\left\{u \in W_{\text {per }}^{1, p}\right.$ $\left.(Y ; \mathbb{R}): \int_{Y} u(y) d y=0\right\}, W_{\mathrm{per}}^{1, p}(Y ; \mathbb{R})$ being the space of $Y$-periodic functions in $W_{\text {loc }}^{1, p}\left(\mathbb{R}^{N} ; \mathbb{R}\right)$. 


\subsection{Problem II}

We plan to study here the homogenization of (1.6) under the hypothesis

$$
f(x, \cdot, \lambda) \in L_{\infty, \mathbb{Z}^{N}}^{1}\left(\mathbb{R}^{N}\right) \text { for all } x \in \bar{\Omega} \text { and all } \lambda \in \mathbb{R}^{n N}
$$

where $L_{\infty, \mathbb{Z}^{N}}^{1}\left(\mathbb{R}^{N}\right)$ denotes the closure in $\left(L^{1}, \ell^{\infty}\right)\left(\mathbb{R}^{N}\right)$ of the space of finite sums

$$
\sum_{\text {finite }} \varphi_{i} u_{i} \text { with } \varphi_{i} \in \mathcal{B}_{\infty}\left(\mathbb{R}^{N}\right), u_{i} \in \mathcal{C}_{\text {per }}(Y),
$$

$\mathcal{B}_{\infty}\left(\mathbb{R}^{N}\right)$ being the space of continuous complex functions on $\mathbb{R}^{N}$ that converge at infinity. Then the appropriate $H$-algebra for this study is $A=\mathcal{B}_{\infty, \mathbb{Z}^{N}}\left(\mathbb{R}^{N}\right)$ where $\mathcal{B}_{\infty, \mathbb{Z}^{N}}\left(\mathbb{R}^{N}\right)$ is defined as in Example 2.3 by taking there $\mathcal{R}=\mathbb{Z}^{N}$. Since $A$ is $W^{1, p}$-proper for any real number $p>1$, the homogenization problem for (1.6) follows under the assumption (4.2).

\subsection{Problem III (Almost periodic setting)}

Before stating the problem to be studied, let us however, recall a general notion of almost periodicity : A function $u \in L_{\mathrm{loc}}^{p}\left(\mathbb{R}^{N}\right)(1 \leq p<\infty)$ is said to be almost periodic in Stepanov sense if $u$ lies in the amalgam space $\left(L^{p}, \ell^{\infty}\right)\left(\mathbb{R}^{N}\right)$ and further the translates $\left\{\tau_{h} u\right\}_{h \in \mathbb{R}^{N}}$ form a relatively compact set in $\left(L^{p}, \ell^{\infty}\right)\left(\mathbb{R}^{N}\right)$. Such functions form a closed vector subspace of $\left(L^{p}, \ell^{\infty}\right)\left(\mathbb{R}^{N}\right)$ denoted by $L_{A P}^{p}\left(\mathbb{R}^{N}\right)$. It is worth recalling that $\left(L^{p}, \ell^{\infty}\right)\left(\mathbb{R}^{N}\right)$ is the space of functions $u \in L_{\text {loc }}^{p}\left(\mathbb{R}^{N}\right)$ such that

$$
\|u\|_{p, \infty}=\sup _{k \in \mathbb{Z}^{N}}\left(\int_{k+(0,1)^{N}}|u(y)|^{p} d y\right)^{\frac{1}{p}}<\infty .
$$

$\left(L^{p}, \ell^{\infty}\right)\left(\mathbb{R}^{N}\right)$ is a Banach space under the norm $\|\cdot\|_{p, \infty}$, and the appropriate norm on $L_{A P}^{p}\left(\mathbb{R}^{N}\right)$ is the $\left(L^{p}, \ell^{\infty}\right)\left(\mathbb{R}^{N}\right)$-norm. It is also worth noting that $A P\left(\mathbb{R}^{N}\right)$ (the space of Bohr almost periodic continuous functions [20]) is a dense vector subspace of $L_{A P}^{p}\left(\mathbb{R}^{N}\right)$. Let $\mathcal{R}$ be a countable subgroup of $\mathbb{R}^{N}$. If we set $L_{A P, \mathcal{R}}^{p}\left(\mathbb{R}^{N}\right)=\left\{u \in L_{A P}^{p}\left(\mathbb{R}^{N}\right): S p(u) \subset \mathcal{R}\right\}$ where $S p(u)=\{k \in$ $\left.\mathbb{R}^{N}: M\left(\bar{\gamma}_{k} u\right) \neq 0\right\}$ with $\gamma_{k}(y)=\exp (2 i \pi k \cdot y)\left(y \in \mathbb{R}^{N}\right)$, then $L_{A P, \mathcal{R}}^{p}\left(\mathbb{R}^{N}\right)$ is a Banach space under the norm $\|\cdot\|_{p, \infty}$, which admits $A P_{\mathcal{R}}\left(\mathbb{R}^{N}\right)=\{u \in$ $\left.A P\left(\mathbb{R}^{N}\right): S p(u) \subset \mathcal{R}\right\}$ as a dense subspace. $A=A P_{\mathcal{R}}\left(\mathbb{R}^{N}\right)$ is an $H$-algebra which is $W^{1, p}$-proper for any real number $p>1$; see Example 2.2.

After these preliminaries, we turn now to the goal of this subsection, which is to study the homogenization problem for (1.6) under the hypothesis

$$
f(x, \cdot, \lambda) \in L_{A P}^{1}\left(\mathbb{R}^{N}\right) \quad \text { for any } x \in \bar{\Omega} \text { and any } \lambda \in \mathbb{R}^{n N} .
$$

But thanks to Corollary 4.1 in [22] we get the existence of a countable subgroup $\mathcal{R}$ of $\mathbb{R}^{N}$ such that

$$
f(x, \cdot, \lambda) \in L_{A P, \mathcal{R}}^{1}\left(\mathbb{R}^{N}\right) \quad \text { for any } x \in \bar{\Omega} \text { and any } \lambda \in \mathbb{R}^{n N} .
$$

Therefore the suitable $H$-algebra for our study here is $A=A P_{\mathcal{R}}\left(\mathbb{R}^{N}\right)$, and in view of Proposition 2.3 the conclusion of Theorem 3.1 is achieved under hypothesis (4.3). 
Remark 4.1. In almost all the previous papers dealing with deterministic homogenization theory (see for instance $[21-23,25]$ ) the almost periodic homogenization problem were stated by combining hypothesis (4.3) above with the following one:

For any $x \in \bar{\Omega}$ and for any $\Psi \in A P\left(\mathbb{R}^{N} ; \mathbb{R}\right)^{n N}$,

$$
\begin{aligned}
& \sup _{k \in \mathbb{Z}^{N}} \int_{k+(0,1)^{N}}|f(x, y-r, \Psi(y))-f(x, y, \Psi(y))| d y \rightarrow 0 \\
& \text { as }|r| \rightarrow 0 .
\end{aligned}
$$

This being so, we observe that here we have two significant improvements: (1) firstly, the hypothesis (4.4) on the uniform equicontinuity is purely dropped; (2) secondly, the homogenization problem is stated here in general terms since the $H$-algebra is $W^{1, p}$-proper for any real $p>1$ and not only for $p=2$ as considered in the papers $[21-23,25]$. This is a true advance as the applications in the almost periodic setting are concerned.

\subsection{Problem IV}

Let $A P\left(\mathbb{R}^{N-1}\right)$ be the space of all continuous complex almost periodic functions on $\mathbb{R}^{N-1}$ (the space of all variables $y^{\prime}=\left(y_{1}, \ldots, y_{N-1}\right)$ ). Let $\mathcal{B}_{\infty}$ $\left(\mathbb{R} ; A P\left(\mathbb{R}^{N-1}\right)\right)$ denote the space of all continuous complex functions $u: \mathbb{R} \rightarrow$ $A P\left(\mathbb{R}^{N-1}\right)$ such that $u\left(y_{N}\right)$ converges in $A P\left(\mathbb{R}^{N-1}\right)$ (with the sup norm) when $\left|y_{N}\right| \rightarrow+\infty$.

We mean to study the homogenization of (1.6) under the hypothesis

$$
f(x, \cdot, \lambda) \in \mathcal{B}_{\infty}\left(\mathbb{R} ; A P\left(\mathbb{R}^{N-1}\right)\right) \text { for all } x \in \bar{\Omega} \text { and all } \lambda \in \mathbb{R}^{n N} .
$$

Arguing as in [22] we see that there exists a countable subgroup $\mathcal{R}^{\prime}$ of $\mathbb{R}^{N-1}$ such that

$$
f(x, \cdot, \lambda) \in \mathcal{B}_{\infty}\left(\mathbb{R} ; A P_{\mathcal{R}^{\prime}}\left(\mathbb{R}^{N-1}\right)\right) \quad \text { for all } x \in \bar{\Omega} \text { and all } \lambda \in \mathbb{R}^{n N} .
$$

It can be shown that $A=\mathcal{B}_{\infty}\left(\mathbb{R} ; A P_{\mathcal{R}^{\prime}}\left(\mathbb{R}^{N-1}\right)\right)$ is a $W^{1, p}$-proper $H$-algebra (indeed $A$ is translation invariant and satisfies all the requirements of [9]) so that the homogenization of (1.6) is achieved under assumption (4.5).

\subsection{Problem V (Perturbed almost periodic homogenization)}

The problem to be studied here states as follows: homogenize the variational minimization problem (1.6) under the assumption

$$
f(x, \cdot, \lambda) \in L_{\infty, A P}^{1}\left(\mathbb{R}^{N}\right) \text { for all } x \in \bar{\Omega} \text { and all } \lambda \in \mathbb{R}^{n N}
$$

where $L_{\infty, A P}^{1}\left(\mathbb{R}^{N}\right)$ denotes the closure in $\left(L^{1}, \ell^{\infty}\right)\left(\mathbb{R}^{N}\right)$ of the space of finite sums

$$
\sum_{\text {finite }} \varphi_{i} u_{i} \text { with } \varphi_{i} \in \mathcal{B}_{\infty}\left(\mathbb{R}^{N}\right), u_{i} \in A P\left(\mathbb{R}^{N}\right) .
$$

Then Arguing as in [22] we deduce the existence of a countable subgroup $\mathcal{R}$ of $\mathbb{R}^{N}$ such that

$$
f(x, \cdot, \lambda) \in L_{\infty, \mathcal{R}}^{1}\left(\mathbb{R}^{N}\right) \quad \text { for all } x \in \bar{\Omega} \text { and all } \lambda \in \mathbb{R}^{n N}
$$


where $L_{\infty, \mathcal{R}}^{1}\left(\mathbb{R}^{N}\right)$ stands for the closure in $\left(L^{1}, \ell^{\infty}\right)\left(\mathbb{R}^{N}\right)$ of the space $A=$ $\mathcal{B}_{\infty, \mathcal{R}}\left(\mathbb{R}^{N}\right)$ defined in Example 2.3, a $W^{1, p}$-proper $H$-algebra for any real number $p>1$. Therefore having in mind Proposition 2.3, we see that the conclusion of Theorem 3.1 follows under hypothesis (4.6).

Other concrete assumptions can be considered, notably the following hypotheses lead to the homogenization of (1.6) for suitable $W^{1, p}$-proper $H$ algebras that can easily be determined:

$(\mathrm{SH})_{1} f(x, \cdot, \lambda)$ is a piecewise continuous function, i.e.,

$f(x, y, \lambda)=c(x, k, \lambda)$ for almost all $y \in k+Y\left(Y=\left(-\frac{1}{2}, \frac{1}{2}\right)^{N}\right), k \in \mathbb{Z}^{N}$,

where $c: \bar{\Omega} \times \mathbb{Z}^{N} \times \mathbb{R}^{n N}$ is a function with the properties $\left(\mathrm{H}_{1}\right)-\left(\mathrm{H}_{4}\right)$, and satisfy the following structure hypothesis

$$
c(x, \cdot, \lambda) \in \mathcal{B}_{\infty}\left(\mathbb{Z}^{N}\right) \quad \text { for all } x \in \bar{\Omega} \text { and all } \lambda \in \mathbb{R}^{n N},
$$

where $\mathcal{B}_{\infty}\left(\mathbb{Z}^{N}\right)$ is the set of all $u: \mathbb{Z}^{N} \rightarrow \mathbb{C}$ such that $\lim _{|k| \rightarrow \infty} u(k) \in \mathbb{C}$.

$(\mathrm{SH})_{2} f(x, \cdot, \lambda) \in \mathcal{B}_{\infty}\left(\mathbb{R} ; L_{\text {per }}^{1}\left(Y^{\prime}\right)\right)$ for all $x \in \bar{\Omega}$ and all $\lambda \in \mathbb{R}^{n N}$, with $Y^{\prime}=$ $(0,1)^{N-1}$.

\subsection{Conclusion}

We have just shown that deterministic homogenization theory also tackle variational minimization problems. It is worth recalling that in the periodic setting, problem (1.6) has been extensively studied. Here we make a brief comparison between the already existing results and ours. In [1] Allaire considers the periodic homogenization of functionals of the form $\int_{\Omega} f(x / \varepsilon, D v(x)) d x$, the integrand $f$ being convex and of class $\mathcal{C}^{1}$ in its second argument. In [2], problem (1.6) is considered but with the continuity hypothesis with respect to the microscopic variable $y$, restricting by this the scope of applications of their results. In the above two works, the connection with our work is that they use the two-scale convergence method to derive their results. In [3] Barchiesi use the multi-scale Young measures to recover our results in the periodic framework. Here we just lay emphasis on the fact that our results also apply in the periodic setting as a particular case. In the almost periodic setting, our results are new. In fact, till now the only results in that setting pertain to Braides [6] and to Kozlov [15] who considered, for the first author, the almost periodic homogenization of functionals of the same type as Allaire (see here above) with $f$ being almost periodic in its first occurrence, and for the second one, the almost periodic homogenization of functionals of the same type, but with $f$ being a quadratic form in its second occurrence. Apart from these two problems (the periodic and the almost periodic ones) all the other problems involved in this study lead to new results. Equally, the study carried out in this paper can easily be generalized to the homogenization of the integral functionals of the form

$$
\int_{\Omega} f\left(x, \frac{x}{\varepsilon}, \frac{x}{\varepsilon^{2}}, D u_{\varepsilon}\right) d x
$$


where the integrand $f$ is convex in its last argument, and to a certain extent, to functionals of the form (1.1) but with $f$ not necessarily convex in its last argument. It would also be interesting to know whether our main result applies to a more range of physical behaviours such as the case of weakly almost periodic [13] environment. Indeed the algebra of weakly almost periodic functions is not separable since it can be shown that the subalgebra of the algebra of weakly almost periodic functions consisting of functions with quadratic mean value zero, is not separable. However, it seems that Theorem 3.1 applies with that algebra. This is an open problem.

\section{Acknowledgments}

The authors would like to thank the anonymous referee for its valuable remarks and comments. The work of the third author is partially supported by a postdoctoral fellow from the University of Pretoria. The support of National Research Foundation of South Africa is acknowledged.

\section{References}

[1] Allaire, G.: Homogenization and two-scale convergence. SIAM J. Math. Anal. 23, 1482-1518 (1992)

[2] Baia, M., Fonseca, I.: $\Gamma$-convergence of functionals with periodic integrands via 2-scale convergence, Technical Report (2005)

[3] Barchiesi, M.: Multiscale homogenization of convex functionals with discontinuous integrand. J. Convex Anal. 14, 205-226 (2007)

[4] Bourbaki, N.: Intégration, Chap. 1-4. Hermann, Paris (1966)

[5] Braides, A.: Homogenization of noncoercive integrals. Ric. Mat. 32, 347-368 (1983)

[6] Braides, A.: Homogenization of some almost periodic coercive functional. Rend. Accad. Naz. Sci. XL 103, 313-322 (1985)

[7] Carbone, L., Sbordone, C.: Some properties of $\Gamma$-limits of integral functionals. Ann. Mat. Pura Appl. 122, 1-60 (1979)

[8] Casado Diaz, J., Gayte, I.: A derivation theory for generalized Besicovitch spaces and its application for partial differential equations. Proc. R. Soc. Edinb. A 132, 283-315 (2002)

[9] Casado Diaz, J., Gayte, I.: The two-scale convergence method applied to generalized Besicovitch spaces. Proc. R. Soc. Lond. A 458, 2925-2946 (2002)

[10] Castaings, C., Valadier, M.: Convex Analysis and Measurable Multifunctions. Lecture Notes in Math., vol. 580. Springer, Berlin (1977)

[11] Champion, T., De Pascale, L.: Homogenization of Dirichlet problems with convex bounded constraints on the gradient. Zeit. Anal. Anw. 22, 591-608 (2003) 
[12] De Giorgi, E., Franzoni, T.: Su un tipo di convergenza variazionale. Atti Accad. Naz. Lincei Rend. Cl. Sci. Mat. Nat. 58, 842-850 (1975)

[13] Eberlein, W.F.: Abstract ergodic theorems and weak almost periodic functions. Trans. Am. Math. Soc. 67, 217-240 (1949)

[14] Fournier, J.J.F., Stewart, J.: Amalgams of $L^{p}$ and $\ell^{q}$. Bull. Am. Math. Soc. 13, $1-21(1985)$

[15] Kozlov, S.: Averaging of differential operators with almost periodic rapidly oscillating coefficients. Math. USSR Sbornik 35, 481-498 (1979)

[16] Larsen, R.: Banach Algebras. Marcel Dekker, New York (1973)

[17] Marcellini, P.: Periodic solutions and homogenization of nonlinear variational problems. Ann. Mat. Pura Appl. 117, 139-152 (1978)

[18] Nguetseng, G.: Homogenization structures and applications I. Zeit. Anal. Anw. 22, 73-107 (2003)

[19] Nguetseng, G.: Mean value on locally compact abelian groups. Acta Sci. Math. 69, 203-221 (2003)

[20] Nguetseng, G.: Almost periodic homogenization: asymptotic analysis of a second order elliptic equation. Preprint (2000)

[21] Nguetseng, G., Nnang, H.: Homogenization of nonlinear monotone operators beyond the periodic setting. Electron. J. Diff. Equ. 2003, 1-24 (2003)

[22] Nguetseng, G., Woukeng, J.L.: Deterministic homogenization of parabolic monotone operators with time dependent coefficients. Electron. J. Diff. Equ. 2004, 1$23(2004)$

[23] Nguetseng, G., Woukeng, J.L.: $\Sigma$-convergence of nonlinear parabolic operators. Nonlinear Anal. TMA 66, 968-1004 (2007)

[24] Van Tiel, J.: Convex Analysis: An Introduction Text. Wiley, New York (1984)

[25] Woukeng, J.L.: Deterministic homogenization of non-linear non-monotone degenerate elliptic operators. Adv. Math. 219, 1608-1631 (2008)

G. Nguetseng

Department of Mathematics,

University of Yaounde 1,

P.O. Box 812,

Yaounde, Cameroon

e-mail: nguetseng@uy1.uninet.cm

H. Nnang

École Normale Supérieure,

P.O. Box 47, Yaounde,

Cameroon

e-mail: hnnang@yahoo.fr 


\section{J. L. Woukeng}

Department of Mathematics and Computer Science, University of Dschang,

P.O. Box 67, Dschang, Cameroon

e-mail: jwoukeng@yahoo.fr

Present Address:

J. L. Woukeng

Department of Mathematics and Applied Mathematics, University of Pretoria, Pretoria 002, South Africa

Received: 09 October 2009.

Revised: 21 May 2010.

Accepted: 07 June 2010. 